\title{
RECENT DEVELOPMENTS AROUND PARTIAL ACTIONS
}

\author{
M. DOKUCHAEV
}

Dedicated to Antonio Paques on the occasion of his 70th birthday

\begin{abstract}
We give an overview of publications on partial actions and related concepts, paying main attention to some recent developments.
\end{abstract}

Since the publication of our previous survey [116] the activity around partial actions enjoyed an increasing intensity, resulting in remarkable applications and theoretic development, as well as the publication of the book [155] by Ruy Exel. The latter contains algebraic and $C^{*}$-algebraic background, a comprehensive treatment of graded $C^{*}$-algebras via Fell bundles and partial $C^{*}$-crossed products, as well as prominent applications to Wiener-Hopf $C^{*}$ algebras associated to quasi-lattice ordered groups and graph $C^{*}$-algebras. The working team gained new participants, in particular, a number of young researchers have been brought to the area by their supervisors. Dedicating this survey to Antonio Paques on the occasion of his 70th birthday, I take the opportunity to register his remarkable leadership in introducing junior scientists into the subject.

For a reader not familiar with our topic, we recall that partial actions appear naturally restricting usual (global) actions as follows. Let $\beta$ be a global action of a group $G$ on a set $Y$, i.e. we have a homomorphism $\beta$ : $G \ni g \mapsto \beta_{g} \in S(Y)$, from $G$ into the symmetric group $S(Y)$ of all bijections (permutations) $Y \rightarrow Y$. Now, let $X \subseteq Y$ be a subset. For each $g \in G$ denote

$$
X_{g}=\beta_{g}(X) \cap X,
$$

and restricting $\beta_{g}$ to $X_{g^{-1}}$ we obtain a bijection $\alpha_{g}: X_{g^{-1}} \rightarrow X_{g}$ between subsets of $X$ (partial bijections of $X$ ). Then the collection $\alpha=\left\{\alpha_{g}: g \in G\right\}$ gives us a partial action of $G$ on $X$. Notice that obviously

(i) $\alpha_{1}=\operatorname{id}_{X}$,

and for all $x \in X, g, h \in G$ :

2000 Mathematics Subject Classification. Primary 16W22, 16S35, 20C99, 46L55; Secondary 08A02, 13B05, 16S10, 16S36, 16T05, 16T15, 16W50, 20C15, 20C25, 20F10, 20L05, 20M18, 20M25, 20M30, 20M50, 22A22, 46L05, 54H15.

Key words and phrases. Partial action, partial representation, crossed product.

This work was partially supported by CNPq and Fapesp of Brazil. 
(ii) $\exists \alpha_{h}(x), \exists \alpha_{g}\left(\alpha_{h}(x)\right) \Longrightarrow \exists \alpha_{g h}(x)$ and $\alpha_{g}\left(\alpha_{h}(x)\right)=\alpha_{g h}(x)$.

Thus one defines a partial action $\alpha$ of a group $G$ on a set $X$ as a family of partial bijections $\alpha_{g}: X_{g^{-1}} \rightarrow X_{g},(g \in G)$ of $X$, such that (i) and (ii) are satisfied. The subsets $X_{g}$ are called the domains of $\alpha$, and one refers to the triple $(X, G, \alpha)$ (or, more precisely, to the quadruple $\left(X, G,\left\{X_{g}\right\}_{g \in G},\left\{\alpha_{g}\right\}_{g \in G}\right)$ ) as a partial dynamical system.

If $X$ has some structure, then one imposes appropriate restrictions on the $X_{g}$ and $\alpha_{g}$. In particular, if we define a partial action on a ring or an algebra (or, more generally, a multiplicative semigroup), then we assume that each domain is a two-sided ideal and each $\alpha_{g}$ is an isomorphism of rings (algebras, or semigroups). ${ }^{1}$ If our ring has an involution then the domains should be closed under the involution and each $\alpha_{g}$ is supposed to respect the involution. If $X$ is a topological space and $G$ is a discrete group, then we assume that each $X_{g}$ is an open subset and each $\alpha_{g}$ is a homeomorphism. In this case we say that $(X, G, \alpha)$ is a topological partial dynamical system. The latter term englobes also a partial action of a topological group $G$ on a topological space, for the definition of which more requirements are needed (see [4, Definition 1.1]). When definining a partial action of a discrete group $G$ on a $C^{*}$-algebra one assumes that each domain is a norm closed ideal and each $\alpha_{g}$ is a $*$-isomorphism of algebras. In this case we have a $C^{*}$-algebraic partial dynamical system. The more general definition of a (continuous) partial action of a locally compact group on a $C^{*}$-algebra was given by R. Exel in [149].

A partial action of a group $G$ on an algebra gives rise to two more concepts: that of a partial action of $G$ on a (multiplicative) semigroup (or monoid) and the notion of a partial action of $G$ on a vector space (or, more generally, a $\kappa$-module over a commutative ring $\kappa$ ). In the first case each domain $X_{g}$ is assumed to be a two-sided ideal in the semigroup and every $\alpha_{g}$ is a semigroup isomorphism. In the second case the $X_{g}$ are subspaces ( $\kappa$-submodules) and the $\alpha_{g}$ are $\kappa$-linear isomorphisms.

As we saw above, any global group action on a set $Y$ can be restricted to any subset $X$, resulting in a partial action whose domains are defined by (1). With some structure on $X$ the above procedure works with an appropriate assumption on $Y$. Thus any group action on a topological space can be restricted to an open subset, and any group action on a ring restricts this way to an arbitrary (two-sided) ideal. This suggests an important question: is a given partial action can be seen (after some identifications) as a

\footnotetext{
${ }^{1}$ Notice that in [72] the authors defined a partial group action on an algebra assuming that each $X_{g}$ is a right ideal generated by an idempotent.
} 
restriction of a global one? In the case of the partial actions on sets the answer is always positive [4] (see also [155, Theorem 3.5]), however, as we shall see below, the situation is more complex with the presence of some structure.

The definition of a partial action of a group $G$ on a set $X$ can be reformulated in several ways. Denote by $\mathcal{I}(X)$ the set of all partial bijections of $X$, including the vacuous bijection $\emptyset \rightarrow \emptyset$. One defines the composition of two partial bijections $\varphi$ and $\psi$ of $X$ on the largest possible subset:

$$
\phi \circ \psi: \psi^{-1}(\operatorname{ran} \psi \cap \operatorname{dom} \varphi) \rightarrow \varphi(\operatorname{ran} \psi \cap \operatorname{dom} \varphi) .
$$

The above operation endows $\mathcal{I}(X)$ with a structure of an inverse monoid with zero $\emptyset \rightarrow \emptyset$, called the symmetric inverse semigroup of $X$. Then a partial action $\alpha$ of $G$ on $X$ gives a map $\alpha: G \ni g \mapsto \alpha_{g} \in \mathcal{I}(X)$, and one may wonder which maps $G \rightarrow \mathcal{I}(X)$ correspond to partial actions on $X$.

The first answer was given by R. Exel in [151, Proposition 4.1] relating it to the important concept of a partial representation: a map $\alpha: G \rightarrow \mathcal{I}(X)$ gives a partial action if and only if for all $g, h \in G$ we have

(a) $\alpha_{1}=\operatorname{id}_{X}$

(b) $\alpha_{g^{-1}} \alpha_{g} \alpha_{h}=\alpha_{g^{-1}} \alpha_{g h}$.

In this case $\alpha$ also satisfies

(c) $\alpha_{g} \alpha_{h} \alpha_{h^{-1}}=\alpha_{g h} \alpha_{h^{-1}}$.

The passage between (b) and (c) can be easily performed by applying the inverses of the partial bijections in $\mathcal{I}(X)$. This result leads to the following relevant notion. A map $\alpha: G \rightarrow S$ from $G$ to a monoid $S$ is said to be a (unital) partial homomorphism if $\alpha$ sends $1_{G}$ to $1_{S}$ and satisfies (b) and (c) above. If $S$ is the multiplicative monoid of a unital algebra, then we say that $\alpha$ is a partial representation. This is the most basic relation between partial actions and partial representations, but there are others resulting in a fruitful interaction, both theoretical and practical.

The inverse semigroup $\mathcal{I}(X)$ possesses a natural partial order given by restriction of partial bijections. More generally, every inverse semigroup $S$ has a natural partial order defined by setting $a \leq b,(a, b \in S)$ if and only if there exists an idempotent $e \in S$ with $a=b e$ (see, for example, [219]). It is immediately seen that (ii) above means that the composition $\alpha_{g} \circ \alpha_{h}$ is a restriction of $\alpha_{g h}$, so that one may write $\alpha_{g} \circ \alpha_{h} \leq \alpha_{g h}$, which is clearly a defining property of partial actions. One may easily obtain another one, i.e. $\alpha_{g^{-1}}=\left(\alpha_{g}\right)^{-1}$. Taking this into account, J. Kellendonk and M. V. 
Lawson [204], [205] gave another way to characterize the maps $G \rightarrow \mathcal{I}(X)$ which determine partial actions. To formalize this, recall that a function $\alpha: S \rightarrow T$ between inverse monoids is called a unital premorphism [205] (also referred to as a dual prehomomorphism [220]) if for all $g, h \in S$ the following properties hold:

(1) $\alpha\left(1_{S}\right)=1_{T}$,

(2) $\alpha\left(g^{-1}\right)=\alpha(g)^{-1}$,

(3) $\alpha(g) \alpha(h) \leq \alpha(g h)$.

Then a map $\alpha: G \rightarrow \mathcal{I}(X)$ determines a partial action precisely when $\alpha$ is a unital premorphism.

Here we warn the reader with respect to the use of terminology. By a partial action of a group $G$ on a set $X$ the authors of [205] mean a family of partial bijections $\alpha=\left\{\alpha_{g}: X_{g^{-1}} \rightarrow X_{g}: g \in G\right\}$ such that the corresponding map $\alpha: G \rightarrow \mathcal{I}(X)$ is a non-necessarily unital premorphism, i.e. $\alpha$ satisfies conditions (2) and (3). If $\alpha$ also satisfies (1), then $\alpha$ is called unital in [205]. Nevertheless, we prefer to assume that (1) is always satisfied, and use the term unital in a different sense. Namely, by a unital partial action we mean a partial action of group $G$ on a ring $\mathcal{A}$ such that each domain is a unital ring, i.e. generated by an idempotent which is central in $\mathcal{A}$. These are exactly those partial actions of $G$ on a unital ring $\mathcal{A}$ which are globalizable (see [117]). Moreover, if each domain is an $s$-unital ring, then $\alpha$ is called an s-unital partial action on $\mathcal{A}$ (see [8] and [42]). Such partial actions appeared in the study of the globalization problem for partial group actions on $s$-unital rings in [136].

One should also notice that the term "partial action" of a group was also used in the literature in a close but different sense, and we refer the reader to the survey [116] for details.

As it is was mentioned in many occasions, the formal concept of a partial action (in the sense we are using it) appeared in the theory $C^{*}$-algebras (see [146], [232], [149], [151]), permitting one to endow relevant classes of $C^{*}$ algebras with a general structure of a partial crossed product [147], [148], [150], [160], [264] (see also [155]), and promptly stimulating further use and discussions in the area [3], [4], [1], [150], [152], [157], [158], [264], [271], [272], [273]. Subsequent $C^{*}$-algebraic and topological developments on partial actions were made in [5], [159], [233], [6], [141], [222], [60], [112], [93], [11] and [163]. 
More recent $C^{*}$ and topological advances include the groupoid approach to the enveloping $C^{*}$-algebras associated to partial actions of countable discrete groups on (locally) compact spaces in [156], the use of inverse semigroup expansions to treat $C^{*}$-crossed products by twisted partial actions via twisted global actions of the expansion in [68], the full (respectively, reduced) partial $C^{*}$-crossed product descriptions of full (respectively, reduced) $C^{*}$-algebras of countable $E$-unitary or strongly 0 - $E$-unitary inverse semigroups as well as of tight groupoids of countable strongly 0 - $E$-unitary inverse semigroups in [235], the study of the continuous orbit equivalence for partial dynamical systems and of the partial transformation groupoids with applications to graph $C^{*}$-algebras and semigroup $C^{*}$-algebras in [224], the partial group action approach to produce a Bratteli-Vershik model linked to a minimal homeomorphism between open subsets with finite disjoint complements of the Cantor set in [179], new developments on the globalization problem for partial actions on $C^{*}$-algebras and Hilbert bimodules in [168], the employment of the partial crossed product theory to the investigation of the Cuntz-Li $C^{*}$-algebras related to an integral domain in [61] with a further development in [281] for $C^{*}$-algebras associated with an injective endomorphism of a group with finite cokernel. Moreover, partial crossed products turned out to be useful to deal with $C^{*}$-algebras arising from self-similar graph actions in [162], with $C^{*}$-algebras associated to any stationary ordered Bratteli diagram in [185], as well as with ultragraph $C^{*}$-algebras and related infinite alphabet shifts in [187], [188], [189] and [191]. In addition, in [212], partial coactions of $C^{*}$-bialgebras, in particular of $C^{*}$-quantum groups, on $C^{*}$-algebras were defined and studied, and a globalization result was obtained.

Partial dynamical systems were also applied in [2] to the study of the ideal structure of full and reduced cross-sectional $C^{*}$-algebras of Fell bundles, in [25] to the investigation of $C^{*}$-algebras of dynamical systems of type $(m, n)$, in [74] to relative graph $C^{*}$-algebras, in [118] to $C^{*}$-algebras associated to arbitrary subshifts, in [180] to the study of the ideals and the pure infiniteness of partial $C^{*}$-crossed products, and in [265] to the investigation of groupoids arising from partial semigroup actions and topological higher rank graphs. Furthermore, partial representations and cohomology based on partial actions turned out to be useful in dealing with the separation and intersection properties of ideals in global reduced $C^{*}$-crossed products [206]. In addition, continuous partial actions of Polish groups on Polish spaces, and more generally, of separable metrizable groups on Hausdorff (in the majority of facts metrizable) spaces were studied in [181], [182], [262] and [263]. In particular, Effros' theorem [144] on orbits in Polish spaces under continuous actions of Polish groups is extended in [181] to the context of partial actions.

A remarkable recent application was achieved to paradoxical decompositions in [23], where for a finite bipartite separated graph $(E, C)$ the tame 
graph $C^{*}$-algebra $\mathcal{O}(E, C)$ was introduced and proved to be isomorphic to a partial crossed product of a commutative $C^{*}$-algebra by a finitely generated free group. This permitted the authors of [23] to give a negative answer to an open problem on paradoxical decompositions in a topological setting, posed in [207], [208] and [267].

The first algebraic results on the subject appeared in [151], [120], [204], [275], [91], [276], [205], [89] and [117], including the first algebraic application for tiling semigroups in [204] (with further use in [283]), for E-unitary inverse semigroups in [205], to inverse semigroups and $F$-inverse monoids in [275] and to inverse monoids of Möbius type in [91]. Independently from Exel's definition of a partial action, T. Coulbois [110] used a more restrictive notion, called a pre-action, to deal with the Ribes-Zalesski property $\left(R Z_{n}\right)$ of groups from model theoretic point of view (see also [111]). Furthermore, J.-C. Birget [56] applied the partial action definition of Thompson's groups to study algorithmic problems for them. Since then the algebraic approach is being developed in diverse directions in various levels of generality, including partial actions of Hopf (or, more generally, weak Hopf) algebras [15], [17], [18], [19], [20], [21], [22], [48], [69], [72], [78], [79], [80], [81], [82], [83], [86], [87], [88], [165], [250], [282], semigroups [68], [97], [132], [197], [198], [199], [209] [213], [220], [227], [233], [234], [242], [255], inductive constellations [198], groupoids [37], [40], [41], [42], [43], [178], and, more generally, categories [244]. In particular, further algebraic applications have been found to graded algebras [117], [121], to Hecke algebras [153], to Leavitt path algebras [184], [186], [190], [247], to inverse semigroups [209], [227], to restriction semigroups [97], [213], to automata and machines [132], [234], [242], [255], and to Steinberg algebras [53], [54].

At this point we observe that the term "partial action" of a monoid (or semigroup) is used in two slightly different senses. First M. G. Megrelishvili and L. Schröder in [233] gave the following definition of a partial monoid action, extending the notion of a partial group action: given a monoid $M$ with unit $e$ and a set $X$, a (left) partial action of $M$ on $X$ is a partially defined map $M \times X \rightarrow X,(m, x) \mapsto m \cdot x$, such that, for all $x \in X, u, v \in M$ :

(i) $e \cdot x=x$, for all $x \in X$,

(ii) $\exists v \cdot x, \exists u \cdot(v \cdot x) \Longrightarrow \exists(u v) \cdot x$ and $u \cdot(v \cdot x)=(u v) \cdot x$,

(iii) $\exists v \cdot x, \exists(u v) \cdot x \Longrightarrow \exists u \cdot(v \cdot x)$ and $u \cdot(v \cdot x)=(u v) \cdot x$.

A right partial action is defined symmetrically. The above was called a strong partial action of a monoid in the article [199] by C. Hollings, whereas in his definition of a partial monoid action (sometimes also referred to as a weak partial action) only $(i)$ and $(i i)$ are assumed. Of course, if in the 
definition by C. Hollings (i.e. in $(i)$ and $(i i)$ ), we replace $M$ by a group $G$, we obtain the concept of a partial group action in the form spelled out by J. Kellendonk and M. V. Lawson in [205]. Ignoring above $(i)$ we obtain the notion of a strong partial action of a semigroup, and omitting both $(i)$ ad (iii) we come to that of a (weak) partial semigroup action. The partial monoid actions considered [97], [213] are weak, whereas in [197] both weak and strong partial monoid (or semigroup) actions are discussed. Strong partial monoid actions were used in [132] under the name "preactions" to define the concept of a preautomaton (see also [234], [242], [255]). Notice that the term "partial automaton" is already in use in a larger sense. Observe that there is a third kind of "partial actions" of monoids (or semigroups) called in [197, Definition 3.2] incomplete actions (see also [199, p. 297] and [266, $\S 3])$. The definition of an incomplete action is obtained by replacing (ii) and (iii) above by the following stronger requirement:

$\left(i i^{\prime}\right) \exists v \cdot x, \exists u \cdot(v \cdot x) \Longleftrightarrow \exists(u v) \cdot x$, in which case $u \cdot(v \cdot x)=(u v) \cdot x$,

$x \in X, u, v \in M$. Each incomplete action is a strong partial action, but there are strong partial actions which are not incomplete (see Examples 2 and 3 in [197]). Note, in addition, that the class of weak partial actions also properly contains the class of strong partial actions [199, Example 2.11].

In the definition of a partial action of an inverse semigroup the language of premorphisms is usually adopted. More precisely, in [220] and [68] a partial action of an inverse semigroup $G$ on a set $X$ is defined as an orderpreserving premorphism $G \rightarrow \mathcal{I}(X)$, whereas in [209] an arbitrary premorphism $G \rightarrow \mathcal{I}(X)$ is considered as a partial action. In [68, Definition 2.11] the notion of a partial homomorphism of an inverse semigroup $G$ into a semigroup is defined and it is proved that a map between inverse semigroups is a partial homomorphism if and only if it is an order-preserving premorphism [68, Proposition 3.1]. Thus, similarly to the group case, partial actions of inverse semigroups on sets from [220] and [68] can be characterized as partial homomorphisms of the form $G \rightarrow \mathcal{I}(X)$. Notice that in [227] partial actions of primitive inverse semigroups on sets were considered and applied to $E^{*}$-unitary categorical inverse semigroups.

More generally, weak and strong right partial actions of a left restriction semigroup $S$ on a set $X$ are defined by V. Gould and C. Hollings in [197, Definitions 3.3, 3.4], by adding to the right-handed forms of (ii) and (iii) an additional axiom which takes into account the presence of the unary operation $u \mapsto u^{+}$:

(iv) $\exists x \cdot u \Longrightarrow \exists x \cdot u^{+}$and $x \cdot u^{+}=x$, 
$u \in S, x \in X$. Left restriction semigroups may be viewed as an axiomatization of the partial transformation monoid $\mathcal{P} \mathcal{I}(X)$ (see [197], [196], [200]). The latter is defined as the semigroup of all partial maps of $X$, i.e. nonnecessarily bijective functions of the form $X \supseteq Y \rightarrow Z \subseteq X$. Notice that each left restriction semigroup can be seen as a subsemigroup of the partial transformation monoid $\mathcal{P} \mathcal{I}(X)$ of some set $X$, which is closed under the unary operation of "taking domains", i.e. ${ }^{+}: \varphi \mapsto \operatorname{id}_{\operatorname{dom} \varphi}$ (see [197, Theorem 2.2], [196, Corollary 6.3]). Since every inverse semigroup $G$ is isomorphic to a ${ }^{+}$-closed subsemigroup of $\mathcal{I}(X) \subset \mathcal{P} \mathcal{I}(X)$, it follows that $G$ is a left restriction semigroup. Another example of a left restriction semigroup is given by the Szendrei expansion of a monoid [200, Proposition 3.3]. It was shown in [197, p. 367] that partial actions of an inverse semigroup $G$, in the sense of [220] and [68], are exactly the strong partial actions of $G$ considered as a left restriction semigroup.

One of the relevant problems in the theory is that of the globalization: given a group (semigroup, groupoid etc.) $G$ acting partially on an object $X$, construct an embedding of $X$ into a larger object $Y$ and a global action of $G$ on $Y$, such that the initial partial action can be obtained as a restriction of the global one. ${ }^{2}$ This was studied first in the PhD Thesis [3] (see also [4]) and, independently from [3], [4], in [205] and [276]. Subsequent results were obtained in [27], [38], [102], [117], [122], [136], [156], [169], and more recently in [8], [50], [51], [105], [168], [262] and [263]. The question was also considered for partial semigroup actions in [197], [199], [209], [233], [213], [227], for partial groupoid actions in [178, 41, 42], and around partial Hopf (co)actions in $[15,18,19,21,78,79,80]$.

The importance of the globalization problem lies in the possibility to relate partial actions with global ones and this way try to move from global results to the partial setting, producing more general facts, as well as to obtain applications to the global case in situations in which partial actions appear naturally, as it occurred in [23]. Thus facts about globalization from [4] were used in [237] with respect to $K$-theory of reduced $C^{*}$-algebras of $0-F$-inverse semigroups, in $[225]$ in the $K$-theoretic study of reduced crossed products attached to totally disconnected dynamical systems, and in [26] for partial flows with application to Lyapunov functions. In addition, globalizable partial actions were essential for the development of Galois Theory of partial group actions in [123], for the elaboration of the concept of a partial Hopf (co)action in [72], as well as in a series of ring theoretic and Galois theoretic investigations in [27], [29], [30], [32], [35], [39], [41], [49], [50], [69], [77], [98], [99], [101], [104], [106], [171], [176], [252], [253].

\footnotetext{
${ }^{2}$ Such a global action with a natural additional assumption is called an enveloping action.
} 
Another way to relate partial and global actions was given in R. Exel's paper [151], in which for any group $G$ a semigroup $\mathcal{S}(G)$ was defined by means of generators $\{[g] \mid g \in G\}$ and relations:

$$
\begin{aligned}
{\left[g^{-1}\right][g][h] } & =\left[g^{-1}\right][g h], \\
{[g][h]\left[h^{-1}\right] } & =[g h]\left[h^{-1}\right], \\
{[g]\left[1_{G}\right] } & =[g],
\end{aligned}
$$

$g, h \in G$ (it follows that $\left.\left[1_{G}\right][g]=[g]\right){ }^{3}$ It was proved that $\mathcal{S}(G)$ is an inverse semigroup [151, Theorem 3.4], and the partial actions of $G$ on a set $X$ are in one-to-one correspondence with the (global) actions of $\mathcal{S}(G)$ on $X$ (see [151, Theorem 4.2]). The injective map

$$
G \ni g \mapsto[g] \in \mathcal{S}(G)
$$

is the canonical partial homomorphism, which plays a key role in the above result. Thus, instead of embedding $X$ into a larger set (or object), one "expands" $G$, obtaining a global action on $X$. It directly follows from the Exel's definition of $\mathcal{S}(G)$ that any partial representation of $G$ can be uniquely extended by means of the above mentioned map $G \rightarrow \mathcal{S}(G)$ to a (usual) representation of $\mathcal{S}(G)$ [151, Proposition 2.2].

The inverse semigroup $S(G)$ can be characterized as an expansion of $G$ in the sense of J.-C. Birget, J. Rhodes [57] (see also [58]), as follows. An expansion is defined as a functor $F$ from the category of semigroups into some special category of semigroups which has the property that there is a natural transformation $\eta$ from the functor $F$ to the identity functor such that $\eta_{S}$ is surjective for every semigroup $S$. Amongst the several expansions discussed in [57], the so called prefix expansion is relevant for us. In [279, Proposition 1] M. Szendrei gave a simple and very useful description of the prefix expansion $\operatorname{Pr}(G)$ (also denoted by $\tilde{G}^{R}$ ) of a group $G$, and proved that $\operatorname{Pr}(G)$ is an $F$-inverse semigroup which enjoys a certain universal " $F$-inverse property" [279, Corollary 3]. The latter is a consequence of a more general result $[279$, Theorem 2], which states that $\operatorname{Pr}($.$) is a functor from the cat-$ egory of groups into the category of $F$-inverse semigroups, which is a left adjoint of the functor assigning the greatest group homomorphic image to any $F$-inverse semigroup.

The construction can be applied to any monoid $M$ (or even to any semigroup) and is called the Szendrei expansion of $M$ [175], [197], [198], [199]. In general the Szendrei expansion $\mathrm{Sz}(M)$ of a monoid $M$ differs from the Birget-Rhodes prefix expansion $\operatorname{Pr}(M)$, but $\operatorname{Sz}(G)=\operatorname{Pr}(G)$ if $G$ is a group. M. Szendrei's idea is of high importance for partial actions, and, in oder to recall it, denote by $\mathcal{P}_{1}(M)$ the set of all finite subsets of a monoid $M$

\footnotetext{
${ }^{3}$ The semigroup $\mathcal{S}(G)$ was denoted by $E(G)$ in [129], [130] and [131].
} 
containing 1. Then

$$
\operatorname{Sz}(M)=\left\{(A, x): A \in \mathcal{P}_{1}(M), x \in A\right\},
$$

with the operation given by

$$
(A, x)(B, y)=(A \cup x B, x y) .
$$

If $M$ is a semigroup without identity element, then one adjoins an external 1 and replaces $M$ by $M^{1}=M \cup\{1\}$ in the above definition. J. Kellendonk and M. V. Lawson have shown in [205] that $\mathcal{S}(G)$ is isomorphic to $\mathrm{Sz}(G)$, and, as a consequence, to $\operatorname{Pr}(G)$. It is derived from a universal property of $\operatorname{Pr}(G)$ with respect to premorphisms [205, Theorem 2.4]. Thanks to the isomorphism $\mathcal{S}(G) \cong \operatorname{Pr}(G)$, Exel's definition of $\mathcal{S}(G)$ gives a presentation for $\operatorname{Pr}(G)=\operatorname{Sz}(G)$ in terms of generators and relations.

The above facts turned $\mathcal{S}(G)$ into a highly important tool, especially when dealing with partial projective group representations [129], [130], [131], and the cohomology theory based on partial actions [124], [125], [126], as well as when relating crossed products by partial actions of groups with crossed products by inverse semigroup actions [163]. They were used in [91] to study realizations of $\operatorname{Pr}(G)$ as an inverse monoid of Möbius type related to a partial action of $G$ on a Hausdorff space. Moreover, the expansion method was further developed and used for partial actions of inverse semigroups in [220], [68], of groupoids in [178], [37], [40], of monoids in [199], of restriction semigroups in [197] and of inductive constellations in [198]. In particular, A. Buss and R. Exel gave in [68] a presentation for the generalized prefix expansion $\operatorname{Pr}(G)$ of an inverse semigroup $G$, introduced earlier by M. V. Lawson, S. W. Margolis and B. Steinberg in [220]. It is proved in [68] that twisted partial actions of $G$ on $C^{*}$-algebras correspond to twisted global actions of $\operatorname{Pr}(G)$, and this correspondence preserves $C^{*}$-crossed products.

It became clear already from the results in [3], [4], [205] and [276] that the globalization problem strongly depends on the category under consideration. In particular, globalizations of partial actions on topological spaces always exist, nevertheless, the topological properties of the initial space are not necessarily shared by the space under the global action. According to [4, Example 1.4] there exists a partial group action on a Hausdorff space whose (minimal) globalization acts on a non-Hausdorff space, and, moreover, in [4, Proposition 1.2] a criteria was given for the preservation of the Hausdorff property under globalization. Because of the categorical equivalence between locally compact Hausdorff spaces and commutative $C^{*}$-algebras, this implies that partial actions on $C^{*}$-algebras are not globalizable in general (see Proposition 2.1 in [4] for a criteria of the existence of a globalization of a partial action on commutative $C^{*}$-algebras). On the other hand, it was shown in [4, Theorem 6.1] that they are globalizable "up to Morita equivalence". More precisely, the concept of Morita equivalence of partial 
actions of locally compact groups on $C^{*}$-algebras was introduced and studied in [4] (see also [273] for the case of discrete groups), as well as that of a Morita enveloping action, which is roughly a global action, whose restriction is a partial action Morita equivalent to the initial one. It was shown that Morita equivalent partial actions have (strongly) Morita equivalent reduced $C^{*}$-crossed products. Furthermore, the reduced $C^{*}$-crossed product of a partial action is (strongly) Morita equivalent to that of the Morita enveloping action. Notice that a particular Morita equivalence fact based on a partial action on a commutative $C^{*}$-algebra from [4] was recently related to a result from [211] by the authors of the above mentioned paper [235]. In the latter article a number of important Morita equivalence facts for $C^{*}$-algebras were estabilshed.

Influenced by F. Abadie's paper [4], the abstract ring theoretic analogues of the above mentioned concepts from [4] were defined and studied in [8]. Facts similar to those from [4] were proved in the context of idempotent rings, whose Morita theory was developed in [177]. Moreover, some further Morita theoretic results were also obtained, including the behavior of Morita equivalent partial actions under the passage to matrices of infinite size with finite number of non-zero entries. The latter has no $C^{*}$-algebraic analogue so far, and the treatment heavily depends on the technique worked out in [121] to prove a ring theoretic analogue of a stabilization result for $C^{*}$-algebraic bundles from [149].

The theory in [8] is developed for the so-called regular partial group actions on idempotent rings. This includes all partial actions on $C^{*}$-algebras, as well as all $s$-unital partial actions. Note that the $s$-unital condition on a ring generalizes all kind of unity conditions in ring theory, including the existence of local units. The regularity assumption imposes a mild restriction on the domains of the partial isomorphisms involved in a partial action, more precisely, the intersection of domains are assumed to coincide with their product. This is a suitable constraint since, on one hand, it resolves the discrepancy between the definitions of partial actions given in [117] and [121], and on the other, in almost all investigations on the subject the considered partial group actions on algebras (or rings) are regular, so that this concept provides a sufficiently general framework for the theory.

It is a well-known fact that Morita equivalent commutative rings with 1 are necessarily isomorphic. More generally this holds for non-degenerate idempotent rings, as established in [177]. An analogous result for partial actions was given in [8]: Morita equivalent $s$-unital partial actions of a group $G$ on commutative algebras must be isomorphic. A similar fact for $C^{*}$-algebras was also established in [8]: Morita equivalent partial actions of a discrete group $G$ on commutative $C^{*}$-algebras are necessarily isomorphic. 
The theory of Morita equivalent partial actions on $C^{*}$-algebras from [4] was further developed by introducing the concept of a weak equivalence for arbitrary Fell bundles over locally compact Hausdorff groups [10] and that of their strong equivalence [7]. The weak equivalence captures the relation between a globalizable partial group action $\alpha$ on a $C^{*}$-algebra and its enveloping action $\beta$ : the Fell bundles corresponding to $\alpha$ and $\beta$ are weakly equivalent (see [10, Example 2.21]). More generally, it follows from the results in [4] and [10] that every Fell bundle associated to a partial action is weakly equivalent to the Fell bundle associated to a global action [10, Corollary 5.15]. Furthermore, amenability is preserved under weak equivalence [10] (see also [7]).

The more restrictive notion of a strong equivalence of Fell bundles is a natural generalization of that of a Morita equivalence of partial actions: two partial actions on $C^{*}$-algebras are Morita equivalent if and only if the Fell bundles associated to them are strongly equivalent [7, Corollary 4.9]. One of the main results in [7] asserts that every Fell bundle is strongly equivalent to a semidirect product Fell bundle for a partial action. Consequently, every Fell bundle is weakly equivalent to the semidirect product Fell bundle of a global action. In addition, it is shown in [7, Corollary 4.3] (see also [10, Proposition 4.13]) that weakly equivalent Fell bundles have (strongly) Morita equivalent full and reduced cross-sectional $C^{*}$-algebras. Notice that [217, Proposition 7.1] implies that the reduced $C^{*}$-algebra of a Fell bundle over a discrete group $G$ is Morita equivalent to the reduced crossed product by a global action of $G$. ${ }^{4}$

In the recent article [168] the problem of the existence of an enveloping action for a partial group action on a non-necessarily unital (abstract) ring was investigated and applied to the globalization problem for partial actions on $C^{*}$-algebras and equivalence Hilbert bimodules. More specifically, amongst various facts it was proved that if a partial action $\alpha=\left\{\alpha_{g}: A_{g^{-1}} \rightarrow\right.$ $\left.A_{g}, g \in G\right\}$ of a (discrete) group on a (non-necessarily unital) ring $A$ admits a globalization, then the following condition is satisfied:

For each $(g, a, b) \in G \times A \times A$ there exists $u \in A_{g}$ such that

$$
c u=\alpha_{g}\left(\alpha_{g^{-1}}(c) a\right) b \text { and } u c=\alpha_{g}\left(a \alpha_{g^{-1}}(b c)\right),
$$

for all $c \in A_{g}$ (see [168, Theorem 2]).

Under appropriate non-degeneracy assumptions (which always hold for $C^{*}$ algebras) condition (2) becomes sufficient for the existence of a globalization for $\alpha$.

\footnotetext{
${ }^{4}$ The author thanks Fernando Abadie for drawing his attention to this fact.
} 
For the $C^{*}$ case, let $G$ be an arbitrary topological group and $\alpha$ be a $C^{*}$-partial action of $G$ on a $C^{*}$-algebra $A$. Contrary to the usual practice in $C^{*}$-theory, $G$ is not assumed to be Hausdorff nor locally compact. The topological generality of $G$ aims to understand the effect of the group's topology on the existence of a globalization. Write $G^{\text {dis }}$ regarding $G$ as a discrete group and let $\alpha^{\text {dis }}$ be the $C^{*}$-partial action of $G^{\text {dis }}$ on $A$ given by $\alpha$. Then [168, Corollary 2] means that for the globalization problem the topology of $G$ can be forgotten, i.e. $\alpha$ admits a $C^{*}$-globalization if and only of so does $\alpha^{\text {dis }}$. Moreover, [168, Theorem 5] asserts that $\alpha$ has a $C^{*}$-globalizaton if and only of $\alpha$ admits a ring theoretic globalization. The latter is shown to be equivalent to the condition given by the first equality in (2). As a consequence, it follows using [117, Theorem 4.5] that a $C^{*}$-partial action $\alpha$ on a unital $C^{*}$-algebra $A$ possesses a $C^{*}$-globalization if and only if $\alpha$ is unital, spelling out thus a $C^{*}$-version of $[117$, Theorem 4.5].

The above mentioned Morita theory for partial actions on $C^{*}$-algebras [4] involves the so-called $C^{*}$-ternary rings, which also may be seen as equivalence Hilbert bimodules, and the author of [168] proves that a partial action of a topological group on an equivalence Hilbert bimodule has a globalization if and only if its linking partial action [4] has a $C^{*}$-globalization [168, Corollary 6]. Notice that new applications of $C^{*}$-ternary rings to $C^{*}$-algebras were given in [9].

Another recent development on the globalization problem was obtained in [21] with respect to twisted Hopf partial actions. Partial actions of Hopf algebras on algebras were defined by S. Caenepeel and K. Janssen in [72] influenced by the Galois theory of partial group actions developed in [123]. The latter stimulated also further Galois theoretic results in [69], which were based on a coring $\mathcal{C}$ constructed for a unital partial action of a finite group, offering thus a more conceptual approach to partial Galois theory via Galois corings. ${ }^{5}$ The coring $\mathcal{C}$ was shown to fit the general theory of cleft bicomodules in [65], and, in addition, in [66] descent theory for corings was applied, using $\mathcal{C}$, to define non-Abelian Galois cohomology $(i=0,1)$ for unital partial Galois actions of finite groups. The article by S. Caenepeel and K. Janssen [72], in its turn, became the starting point for a series of investigation of partial Hopf (co)actions (see the articles cited above), in particular, several globalization results were obtained in [15], [18], [19], [78], [79], [80] and [212].

On the other hand, the twisted version of partial group actions on (abstract) algebras were introduced and studied in [121]. This was inspired by the R. Exel's notion of a continuous twisted partial action of a locally compact group on a $C^{*}$-algebra (a twisted partial $C^{*}$-dynamical system) and

\footnotetext{
${ }^{5}$ The authors of [69] use the term idempotent partial action, nevertheless we prefer to employ the latter name in a different sense.
} 
that of the corresponding crossed product [149]. The new construction permitted one to show that any second countable $C^{*}$-algebraic bundle, which satisfies a certain regularity condition (automatically verified if the unit fiber algebra is stable), is a $C^{*}$-crossed product of the unit fiber algebra by a continuous partial action of the base group [149]. The algebraic version of the latter fact was established in [121]. This algebraic concept was applied to Hecke algebras in [153], where, among other results, it was proved that given a field $\kappa$ of characteristic 0 , a group $G$ and subgroups $H, N \subseteq G$ with $N$ normal in $G$ and $H$ normal in $N$, there is a twisted partial action $\theta$ of $G / N$ on the group algebra $\kappa(N / H)$ such that the Hecke algebra $\mathcal{H}(G, H)$ is isomorphic to the crossed product $\kappa(N / H) *_{\theta} G / N$. The globalization problem for twisted partial group actions was investigated in [122], whereas other algebraic results on twisted partial actions on rings and corresponding crossed products were obtained in [39], [44], [49], [50] and [253].

Motivated by the concept of a twisted partial group action given in [121] on one hand, and the developments on partial Hopf actions on the other, twisted partial actions of Hopf algebras on rings were introduced in [20], as well as the corresponding crossed products. Examples using algebraic groups were elaborated, more precisely, actions of an affine algebraic group on affine varieties give rise to coactions of the corresponding commutative Hopf algebra $H$ on the coordinate algebras of the varieties, restrictions of which produce concrete examples of partial Hopf coactions. Then one dualizes in order to obtain partial Hopf actions. The dualization passage works theoretically, but may far from being easy in concrete examples. One possibility is to try to identify the finite dual $H^{0}$ for a specific $H$ obtained in the above way. A more flexible possibility is to find a concrete Hopf algebra $H_{1}$ such that $H$ and $H_{1}$ form a dual pairing. Then [18, Proposition 8] produces a partial action of $H_{1}$. A concrete example was elaborated [20], which was easily endowed with a twisted structure by means of a 2-cocycle. Furthermore, symmetric twisted partial Hopf actions were introduced in [20] in order to treat the convolution invertibility of the partial cocycle in a manageable way. This permitted one to get a relation between crossed products and the so-called partially cleft extensions. The latter were also introduced in the same paper [20], and the definition reflects "partiality" in more than one way, incorporating, in particular, some equalities already proved to be significant in the study of partial group actions and partial representations. Then one of the main facts in [20] states that the partial cleft extensions over the coinvariants $A$ are exactly the crossed products by symmetric twisted partial Hopf actions on $A$. One should notice that it was proved in [165] that the crossed products by twisted partial Hopf actions [20] form a particular case of the more general weak crossed products defined in [164].

Then it became natural to investigate the globalization problem for twisted partial Hopf actions on rings [21]. The main result says that a symmetric 
twisted partial action of a Hopf algebra $H$ on a unital algebra $\mathcal{A}$ associated to the symmetric pair of partial cocycles $\omega$ and $\omega^{\prime}$, is globalizable if, and only if, there exists a normalized convolution invertible linear map $\tilde{\omega}: H \otimes H \rightarrow \mathcal{A}$ satisfying certain compatibility conditions, intertwining the partial action of $H$ on $\mathcal{A}$ and the restriction of the twisted action of $H$ on $\mathcal{B}$. A series of examples were elaborated. In particular, a complete characterization of partial measuring maps were given in the case of $H$ being the group algebra $\kappa G$, the dual $(\kappa G)^{*}$ of the group algebra $\kappa G$ of a finite group $G$, and the Sweedler Hopf algebra $H_{4}$, and $\mathcal{A}$ coinciding (in all three cases) with the base field $\kappa$. Furthermore, symmetric twisted partial Hopf actions were described in details for specific Hopf algebras. In particular, the case of a group algebra $\kappa G$ recovers the theory of twisted partial actions of groups as developed in [121] and [122]. For the Sweedler Hopf algebra $H_{4}$, the only symmetric twisted partial actions are the global ones. In addition, for the case of the dual $(\kappa G)^{*}$ of the group algebra of a finite group $G$, the partial appearing cocycles have remarkable symmetries, and they were related to global cocycles of dual group algebras of quotient groups. The specific example for the Klein four-group $K_{4}$, acting on the base field $\kappa$, was done in more details, and it was shown that the symmetric twisted partial actions of $\left(\kappa K_{4}\right)^{*}$ on $\kappa$ are parametrized by the zeros $(x, y) \in \kappa^{2}$ of a polynomial in $x, y$ of degree 2 . An explicit partial cocycle for $\left(\kappa K_{4}\right)^{*}$ was given which leads to a globalizable symmetric twisted partial action. Moreover, it was shown that the example of a twisted partial Hopf action, constructed in [20] using the relation between algebraic groups and Hopf algebras, is globalizable.

The algebraic advances on Hopf partial actions influenced a $C^{*}$-theoretic development: in the already mentioned paper [212], the approach for partial Hopf coactions from [19] motivated a globalization result for partial coactions of $C^{*}$-quantum groups satisfying a mild restriction, which always holds if the quantum group is discrete, or if the $C^{*}$-algebra of the quantum group is nuclear. The construction of the enveloping action gives a left adjoint to the forgetful functor from coactions to partial coactions (with an appropriate choice of categories).

The algebraic concept of twisted partial actions also motivated the study of projective partial group representations, the corresponding partial Schur Multiplier and the relation to partial group actions with $\kappa$-valued twistings in [129], [130] and [131], and contributing thus towards the elaboration of a background for a general cohomology theory based on partial actions.

A (usual) projective representation of a group $G$ can be defined as a homomorphism from $G$ to the projective linear group $\mathrm{PGL}_{n}(\kappa)$. In order to define partial projective representations one replaces usual homomorphisms by partial ones as follows. Denote by $\mathrm{PMat}_{n} \kappa$ the monoid of the projective $n \times n$ matrices over a field $\kappa$, i.e. $\operatorname{PMat}_{n} \kappa=\left(\operatorname{Mat}_{n} \kappa\right) / \lambda$, where $\lambda$ is the 
congruence given by $A \lambda B \Longleftrightarrow A=c B$ for some $c \in \kappa^{*}$. Then we define a partial projective representation of $G$ as a partial homomorphism of the form $G \rightarrow$ PMat $_{n} \kappa$ [129]. Taking representatives in the congruence classes, we may consider a partial projective representation as a function of the form $G \rightarrow \operatorname{Mat}_{n} \kappa$. As in the classical case, factor sets appear naturally: a factor set of a partial projective representation $\Gamma: G \rightarrow$ Mat $_{n} \kappa$ is a function $\sigma: G \times G \rightarrow \kappa$ such that

$$
\begin{gathered}
\sigma(g, h)=0 \Longleftrightarrow \Gamma(g) \Gamma(h)=0, \\
\Gamma\left(g^{-1}\right) \Gamma(g) \Gamma(h)=\Gamma\left(g^{-1}\right) \Gamma(g h) \sigma(g, h),
\end{gathered}
$$

and

$$
\Gamma(g) \Gamma(h) \Gamma\left(h^{-1}\right)=\Gamma(g h) \Gamma\left(h^{-1}\right) \sigma(g, h),
$$

for all $g, h \in G$ (see [129, Theorem 3]). The set

$$
X=X_{\sigma}=\{(g, h) \in G \times G: \Gamma(g) \Gamma(h) \neq 0\}
$$

is called the domain of $\sigma$ (or the domain of $\Gamma$ ).

More generally, in [129] the partial projective representations are considered as functions of the form $\Gamma: G \rightarrow M$, where $M$ is a so-called $\kappa$-cancellative monoid (see [129, Definition 2]).

The theory of partial projective representations is strongly related to Exel's semigroup $\mathcal{S}(G)$. In fact, they can be alternatively defined via projective representations of $\mathcal{S}(G)$, so that the theory of projective representations of semigroups and their Schur multipliers, elaborated by B. Novikov in [238], [239], [240] (see also [241]), comes into the picture as an essential working tool. The usual cohomology of semigroups does not serve the projective semigroup representations, instead the more general 0-cohomology [240] fits them with its natural partial flavor.

The factor sets of the partial projective representations of $G$ form a commutative semigroup, whose equivalence classes constitute the partial Schur Multiplier $p M(G)$. The latter is a commutative inverse semigroup, and as such it is a semilattice of abelian groups $p M_{X}(G)$, called components, where $X$ runs over the domains of the partial projective representations of $G$. One of the component, namely, the group of the equivalence classes of the totally defined factor sets $p M_{G \times G}(G)$, contains the usual Schur Multiplier $M(G)$ of $G$, but $p M_{G \times G}(G)$ is essentially bigger than $M(G)$. In [130] the structure $p M_{G \times G}(G)$ was investigated over an algebraically closed field $\kappa$ and the technique of [130] was further developed in [131], permitting one to extend the description to any component $p M_{X}(G)$, and to show that each $p M_{X}(G)$ is an epimorphic image of $p M_{G \times G}(G)$. Furthermore, it was also shown in [131] that each $p M_{X}(G)$ is an epimorphic image of a direct power of $\kappa^{*}(\kappa$ 
is assumed to be algebraically closed).

Since the partial projective representations of a group $G$ are intimately related to the projective representations of the inverse semigroup $\mathcal{S}(G)$, it was natural to explore this connection and use it as a working tool. This relation was established in [129] and further explored in [130] and [131], incorporating some simplifications. One of them is a passage from the semigroup $\mathcal{S}(G)$ to its quotient $\mathcal{S}_{3}(G)$. In order to define the latter, we identify $\mathcal{S}(G)$ with $\operatorname{Sz}(G)$, sending the generator $[g] \in \mathcal{S}(G), g \in G$, from the initial definition of $\mathcal{S}(G)$ to the pair $(\{1, g\}, g) \in \mathrm{Sz}(G)$, [205]. Denoting by $N_{k}$ the ideal $\{(R, g) \in \mathcal{S}(G)|| R \mid \geq k+1\}$ of $\mathcal{S}(G)$, the semigroup $\mathcal{S}_{3}(G)$ is the quotient $\mathcal{S}(G) / N_{3}(G) .{ }^{6}$ Reductions based on this passage permitted one to obtain characterizations of partial factor sets of $G$ over an algebraically closed field, one of which gives defining equalities for the partial factor sets that curiously have nothing to do with the 2-cocycle identity, but nicely incorporate the symmetry under the action of the symmetric group $S_{3}$ [131, Theorem 5.6]. These results led to general facts on the structure of the components of $p M(G)$ and allowed one to perform calculations for concrete groups.

R. Exel's concept of a continuous twisted partial action [149] and its purely ring theoretic version [121] involve a general twisting which satisfies the 2-cocycle identity in some restricted sense, and it was natural to fit this in some cohomology theory. A relation of this cohomology to the partial Schur Multiplier is naturally expected, as the classical Schur Multiplier is isomorphic to the cohomology group $H^{2}(G, \mathbb{C})$, where the action of $G$ on $\mathbb{C}$ is trivial. The main idea is to replace usual $G$-modules, i.e. global actions of $G$ on abelian groups, by partial $G$-modules, which are partial actions of $G$ on commutative monoids. The first step was done in [124] with more recent developments in the preprints [125] and [126]. Since the twistings in [121] take values in multiplier algebras of products of some ideals, it was reasonable to avoid multipliers at the beginning, imposing a rather usual restriction on the partial action, namely, that it is unital. We recall that a partial action on a ring (or a semigroup) is called unital if each domain is an ideal generated by an idempotent, which is central in the ring (semigroup).

If we assume that the $\operatorname{ring} \mathcal{A}$ under a unital twisted partial action of a group $G$ is commutative, then the action falls into two parts: a partial action $\alpha$ of $G$ on $\mathcal{A}$ and its twisting. This way we derive the notion of a partial 2cocycle (the twisting) whose values belong to groups of invertible elements of appropriate ideals of $\mathcal{A}$. The concept of a partial 2-coboundary then follows from that of an equivalence of twisted partial actions introduced in [122]. Of course, in the general definition we do not need a ring structure on $\mathcal{A}$, so

\footnotetext{
${ }^{6}$ The semigroup $\mathcal{S}_{3}(G)$ was denoted by $E_{3}(G)$ in [130] and [131].
} 
assuming that $A$ is a commutative multiplicative monoid, one comes to the definition of the second cohomology group $H^{2}(G, A)$. The groups $H^{n}(G, A)$ with arbitrary $n$ are defined in a similar way. More precisely, given a partial $G$-module $A$, i.e. a commutative monoid endowed with a partial action $\alpha=\left\{\alpha_{g}: A_{g^{-1}} \rightarrow A_{g}, g \in G\right\}$, for any $n>0$ one defines $n$-cochains of $G$ with values in $A$ as functions $f: G^{n} \rightarrow A$, such that $f\left(x_{1}, \ldots, x_{n}\right)$ is an invertible element of the ideal $A_{\left(x_{1}, \ldots, x_{n}\right)}=A_{x_{1}} A_{x_{1} x_{2}} \ldots A_{x_{1} \ldots x_{n}}$. By $a$ 0 -cochain we mean an invertible element of $A$. Then the set $C^{n}(G, A)$ of $n$-cochains is an abelian group under the pointwise multiplication with the identity element

$$
e_{n}\left(x_{1}, \ldots, x_{n}\right)=1_{x_{1}} 1_{x_{1} x_{2}} \ldots 1_{x_{1} \ldots x_{n}},
$$

and the inverse of $f \in C^{n}(G, A)$ being $f^{-1}\left(x_{1}, \ldots, x_{n}\right)=f\left(x_{1}, \ldots, x_{n}\right)^{-1}$, where $f\left(x_{1}, \ldots, x_{n}\right)^{-1}$ means the inverse of $f\left(x_{1}, \ldots, x_{n}\right)$ in $A_{\left(x_{1}, \ldots, x_{n}\right)}$.

Next, for any $f \in C^{n}(G, A)$ and $x_{1}, \ldots, x_{n+1} \in G$ define the coboundary map:

$$
\begin{aligned}
\left(\delta^{n} f\right)\left(x_{1}, \ldots, x_{n+1}\right) & =\alpha_{x_{1}}\left(1_{x_{1}^{-1}} f\left(x_{2}, \ldots, x_{n+1}\right)\right) \\
& \prod_{i=1}^{n} f\left(x_{1}, \ldots, x_{i} x_{i+1}, \ldots, x_{n+1}\right)^{(-1)^{i}} \\
& f\left(x_{1}, \ldots, x_{n}\right)^{(-1)^{n+1}} .
\end{aligned}
$$

Here the inverse elements are taken in the corresponding ideals. If $n=0$ and $a$ is an invertible element of $A$, we set $\left(\delta^{0} a\right)(x)=\alpha_{x}\left(1_{x^{-1}} a\right) a^{-1}$. Then $\delta^{n}$ is a homomorphism $C^{n}(G, A) \rightarrow C^{n+1}(G, A)$, and

$$
\delta^{n+1} \delta^{n} f=e_{n+2}
$$

for any $f \in C^{n}(G, A)$ (see [124]). Now, as in the classical case, we define the abelian groups $Z^{n}(G, A)=\operatorname{Ker} \delta^{n}, B^{n}(G, A)=\operatorname{Im} \delta^{n-1}$ and

$$
H^{n}(G, A)=\operatorname{Ker} \delta^{n} / \operatorname{Im} \delta^{n-1}
$$

of partial $n$-cocycles, $n$-coboundaries and $n$-cohomologies of $G$ with values in $A, n \geq 1\left(H^{0}(G, A)=Z^{0}(G, A)=\operatorname{Ker} \delta^{0}\right)$.

One actually may replace $A$ by an appropriate submonoid $\tilde{A}$, which is inverse [124]. This brings $A$ closer to the classical case, as the commutative inverse monoids are natural generalizations of abelian groups, not being too far from them.

One of the difficulties with the partial $G$-modules is that they do not form an abelian category. Nevertheless, our partial cohomology can be related to the Lausch-Leech-Loganathan cohomology of inverse semigroups (see [218], $[223]$ and [229]) via the R. Exel's inverse monoid $\mathcal{S}(G)$. From a unital partial action of $G$ on $A$ one comes to an action of $\mathcal{S}(G)$ and then to an "almost" 
Lausch's $\mathcal{S}(G)$-module structure on $A$. The latter can be seen as a module in the sense of H. Lausch over an epimorphic image of $\mathcal{S}(G)$, provided that $A$ is an inverse partial $G$-module. Thus our category is made up of abelian "pieces" which are categories of Lausch's modules over epimorphic images of $\mathcal{S}(G)$. This way we are able to define free objects and free resolutions which lead to $H^{n}(G, A)$ [124]. We also showed that the partial Schur multiplier $p M(G)$ is a union of 2-cohomology groups of $G$ with values in non-necessarily trivial partial $G$-modules.

Then it was natural to give an interpretation of the partial 2-cohomology group in terms of extensions. This was initiated in [125], and at some point it became clear that it is preferable to abandon the restriction on a partial action to be unital, imposed by cohomology theory in [124], covering thus a more general situation. The key notion is that of an extension of a semilattice of groups $A$ by a group $G$ [124], the main example being the crossed product $A *_{\alpha} G$ by a twisted partial action $\alpha$ of $G$ on $A$. Such an extension is related to the notion of an extension of $A$ by an inverse semigroup $S$, that of a twisted $S$-module and the corresponding crossed product given in [218]. In particular, given an extension $A \rightarrow U \rightarrow G$ there is a refinement $A \rightarrow U \rightarrow S \rightarrow G$ such that $A \rightarrow U \rightarrow S$ is an extension of $A$ by $S$, where $S$ is an $E$-unitary semigroup. In order to make the theory work well we impose an admissibility condition on the extensions $A \rightarrow U \rightarrow G$, and the $S$-module structures on $A$, that we obtain this way, possesses twistings which satisfy a normality condition, considered by N. Sieben in [272], which is stronger than the one imposed by H. Lausch in [218]. For this reason we call them Sieben's twisted modules. Then we are able to show that any admissible extension $A \rightarrow U \rightarrow G$ is equivalent to some crossed product extension $A \rightarrow A *_{\alpha} G \rightarrow G$, and the final fact in [125] establishes an equivalence preserving one-to-one correspondence between twisted partial actions of groups on $A$ and Sieben's twisted module structures on $A$ over $E$-unitary inverse semigroups.

The theory of extensions in [125] went beyond the cohomology theory in [124], so that it became clear that it is more natural to deal with a more general multiplier valued cohomology theory. This is done in [126] where the cohomology groups $H^{n}(G, A)$, with $A$ being a non-necessarily unital partial $G$-module, were defined. For an inverse semigroup $S$ and an $S$-module structure on $A$ we also define the cohomology groups $H_{\leq}^{n}\left(S^{1}, A^{1}\right)$ based on order preserving cochains and relate them to $H^{n}(G, A)$. This is motivated by the fact that Sieben's twisted $S$-modules have order preserving twistings. The elements of the second cohomology group $H^{2}(G, A)$ are proved to be in one-to-one correspondence with the equivalence classes of the extensions of $A$ by $G$. In addition, we define the concept of a split extension $A \rightarrow U \rightarrow G$ and prove that the elements of $H^{1}(G, A)$ are in one-to-one correspondence 
with the equivalence classes of splittings of $U$.

Note that partial group cohomology turned out to be useful to study ideals of global reduced $C^{*}$-crossed products: to a given global $C^{*}$-dynamical system the authors of [206] associate a partial $C^{*}$-dynamical system, giving rise to a "twisted partial representation", which is a projective partial representation, whose factor set (twist) is a partial 2-cocycle $\sigma$. If $\sigma$ is a 2-coboundary, then the initial (global) $C^{*}$-dynamical system is said to have "vanishing obstruction". As the authors say: "In a certain precise sense, the twist is the only obstruction to understanding the ideal structure of the reduced crossed product". Assuming the vanishing obstruction property, several necessary and sufficient conditions for the ideal intersection property are given. The latter is known to be closely related to the ideal separation property.

Further results on partial projective representations were obtained in [128], [131], [226], [243], [257], [258], [260] and [261] (see also the short survey [259]). In particular, computations of the partial Schur multiplier of concrete groups in [131], [226], [243], [258], [260] show that each component is, in fact, isomorphic to a direct power of $\kappa^{*}$, suggesting that this should be true for all groups. This motivated the recent preprint [137], in which this conjecture was confirmed for all finite groups over an algebraically closed field. This surprisingly gives a better understanding of the structure of $p M(G)$ than one has for that of the usual Schur Multiplier.

Using [129, Proposition 2] one can show that a partial factor set $\sigma$ satisfies the following weak 2-cocycle condition:

$$
1 \in\{x, y, z, x y, y z, x y z\} \Rightarrow \delta^{2} \sigma(x, y, z)=0 .
$$

The key idea in [137] is to replace $\kappa^{*}$ by an arbitrary abelian group $A$ and define pre-cocycles which are functions $\sigma: G \times G \rightarrow A$ obeying condition (6). They form a group denoted by $p Z^{2}(G, A)$. Since the equivalence of partial factor sets is defined modulo classical coboundaries, it is reasonable to introduce the factor group $p Z^{2}(G, A) / B^{2}(G, A)$, which is denoted by $p H^{2}(G, A)$ and called the pre-cohomology group of $G$ with values in $A$. Aiming to generalize the components $p M_{X}(G)$ of the partial Schur multiplier $p M(G)$, one wishes to restrict pre-cocycles to domains. By [131, Proposition 5.3] there is a bijection between the domains of factor sets of partial projective representations of $G$ and the following set of ideals:

$$
\Lambda=\left\{I \unlhd \mathcal{S}(G) \mid N_{3} \leq I \neq \mathcal{S}(G)\right\} .
$$

The correspondence takes a domain $X$ to the ideal $I \in \Lambda$ such that for all $x, y \in G$ :

$$
(x, y) \in X \Longleftrightarrow[x][y] \notin I .
$$


Let now $A \cup\{0\}$ be the semigroup obtained by adjoining a zero 0 to the group $A$ (we assume the multiplicative notation for $A$ ). ${ }^{7}$ Considering for any ideal $I \in \Lambda$ the function $\varepsilon_{I}: G \times G \rightarrow A \cup\{0\}$ determined by

$$
\epsilon_{I}(g, h)=\left\{\begin{array}{cl}
0 & \text { if }[g][h] \in I \\
1_{A} & \text { otherwise }
\end{array}\right.
$$

and setting

$$
Z^{2}(G, I ; A)=p Z^{2}(G, A) \varepsilon_{I}, \quad B^{2}(G, I ; A)=B^{2}(G, A) \varepsilon_{I},
$$

the quotient

$$
H^{2}(G, I ; A)=Z^{2}(G, I ; A) / B^{2}(G, I ; A),
$$

is called the second partial cohomology group relative to $I$ with coefficients in $A$. As the abelian groups $p M_{X}(G)$ assembles into the partial Schur multiplier $p M(G)$, it is natural to define the second partial cohomology semilattice of groups of $G$ by

$$
H^{2}(G, \Lambda ; A)=\coprod_{I \in \Lambda} H^{2}(G, I ; A) .
$$

Another fruitful point in [137] is to view partial factor sets as liftings (sections) $\varphi: G \rightarrow E$ of partial homomorphisms $\psi: G \rightarrow M$ related to appropriate central extensions $\kappa^{*} \rightarrow E \rightarrow M$ fitting the following commutative diagram:

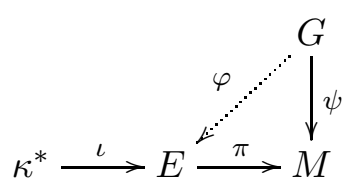

Then for any $\sigma \in Z^{2}(G, I ; A)$ it is possible to construct a central extension $A \rightarrow E \rightarrow M$, a partial homomorphism $\psi: G \rightarrow M$ and its lifting (section) $\varphi: G \rightarrow E$, such that the diagram (7) is commutative upon the replacement of $\kappa^{*}$ by $A$, and $\sigma$ plays the role of the factor set related to $\varphi$ as in (3), (4), (5). This leads to a bijection between the elements of $H^{2}(G, I ; A)$ and the equivalence classes of certain appropriately defined central extensions [137, Theorem 3.3]. This way the partial Schur multiplier $p M(G)$ over an arbitrary field $\kappa$ coincides with the second partial cohomology semilattice of groups with values in $A=\kappa^{*}$, the components of which being second relative partial cohomology groups.

Theorem $4.3 \mathrm{em}$ [137] gives a precise structure of the pre-cohomology group for a finite group $G$ of order $n$ an any abelian group $A$ :

$$
p H^{2}(G, A) \simeq A^{m-n} \oplus A \otimes G /[G, G],
$$

\footnotetext{
${ }^{7} \operatorname{In}[137] A$ is additive and the zero element is denoted by $\infty$.
} 
where $m=\left(n^{2}+2\left|G_{(3)}\right|+3\left|G_{(2)}\right|+5\right) / 6$ and $G_{(k)}$ stands for the set of elements of order $k$ in $G$. Notice that $Z^{2}\left(G, N_{3} ; A\right)=p Z^{2}(G, A)$. The structure result [137, Theorem 5.2] for a general relative cohomology group $H^{2}(G, I ; A)$ is less precise but it is good enough to conclude that if $G$ is finite, $A=\kappa^{*}$ and $\kappa$ is algebraically closed, then each $H^{2}\left(G, I ; \kappa^{*}\right)$ is isomorphic to a finite direct power of $\kappa^{*}$.

The relative partial cohomology is consistent with the partial cohomology in [124]: each second partial cohomology group relative to an ideal is isomorphic to the second partial cohomology group with values in an appropriate unital partial $G$-module [137, Theorem 6.1]. As a consequence, one concludes that for a finite $G$ and an arbitrary field $\kappa$, each component of $p M(G)$ is isomorphic to a single cohomology group with values in some partial $G$-module. The latter fact brings the partial Schur theory closer to the classical one.

While partial group actions and partial group representations are strongly related to inverse semigroups, a generalization of the latter, namely, the (two-sided) restriction semigroups, found interesting applications of partial actions of monoids in [97] and [213]. The notion of a (two-sided) restriction semigroup is based on the existence of two unary operations $u \mapsto u^{+}$ and $u \mapsto u^{*}$, which resemble the maps $x \mapsto x^{-1} x$ and $x \mapsto x x^{-1}$ in an inverse semigroup. Its axiomatic definition incorporates the defining identities of both left and right restriction semigroups, as well as two connecting relations $\left(u^{+}\right)^{*}=u^{+},\left(u^{*}\right)^{+}=u^{*}$ (see [97] or [213]). In particular, every subsemigroup of an inverse semigroup that is closed under ${ }^{+}$and ${ }^{*}$ serves as an example of a restriction semigroup. The role of the relevant class of the $E$-unitary inverse semigroups is played now by the proper restriction semigroups, for which in [97] a structure theorem in terms of double partial actions of monoids on semilattices was given. This extends classical results for inverse and ample semigroups. The double partial action from [97] can be reformulated in terms of only one partial action, and in [213] classes of proper restriction semigroups determined by the properties of this partial action where classified. In particular, a new important class of proper restriction semigroups was introduced this way, which were called ultra proper restriction semigroups, and which nicely relates to other wellestablished classes. The author of [213] uses partial actions to obtain various relevant results, in particular, globalizations of a partial actions are applied to establish a McAlister-type theorem, as well an embedding fact into $W$ products, extending known results, and producing new and simpler proofs.

Another recent application of partial actions to semigroup theory was given in [209], offering a simple proof of a weakened version of an embedding theorem by O'Carroll [249]. The notion of a partial crossed product semigroup (also called partial semidirect product, if the twisting is trivial), 
considered earlier with some variations ${ }^{8}$ in [205], [275], [129], [130], [124] and [125], becomes useful for this purpose: given an idempotent pure congruence $\rho$ on an inverse semigroup $S$, there is a partial action $\tau$ of $S / \rho$ on the semillatice of the idempotents of $S$ such that $S$ embeds into the partial semidirect product determined by $\tau$ (see [209, Theorem 3.4]). Recall that a partial action of an inverse semigroup is defined in [209] by means of an arbitrary premorphism into the symmetric inverse monoid, so that this notion is weaker than that one adopted in [220] and [68], where the premorphism is assumed to be order-preserving. The image of $S$ in the partial semidirect product is specified with the help of the concept of a fully strict partial action of an inverse semigroup on a semilattice. It is also proved, using globalization, that O'Carroll's [249, Theorem 4] is [209, Theorem 3.4] with globalizable $\tau$. It is pointed out by means of an example that $\tau$ is not always globalizable.

Having elaborated the bases of a cohomology theory of partial $G$-modules, one may try to extend the technique from [122] to globalize partial $n$ cocycles. The main result in [122] asserts that an arbitrary unital twisted partial action $\alpha$ of a group $G$ on a (unital) ring $\mathcal{A}$, which is a product of indecomposable rings (blocks), admits an enveloping action, i.e. there exists a twisted global action $\beta$ of $G$ on a ring $\mathcal{B}$ such that $\mathcal{A}$ can be embedded into $\mathcal{B}$ as a two-sided ideal, such that $\alpha$ can be seen as the restriction of $\beta$ to $\mathcal{A}$ and $\mathcal{B}=\sum_{g \in G} \beta_{g}(\mathcal{A})$. Moreover, if $\mathcal{B}$ has $1_{\mathcal{B}}$, then any two globalizations of $\alpha$ are equivalent in a natural sense.

If $\mathcal{A}$ is commutative, then the above mentioned results from [122] mean that given a unital $G$-module structure on $\mathcal{A}$, for any 2-cocycle of $G$ with values in $\mathcal{A}$ there exists a (usual) 2-cocycle $u$ of $G$ related to the global action on $\mathcal{B}$ such that $w$ is the restriction of $u$. Moreover, if $\mathcal{B}$ has $1_{\mathcal{B}}$, then any two globalizations of $w$ are cohomologous.

The proofs in [122] are rather technical. Nevertheless, thanks to some improvements we managed in [127] to extend to arbitrary $n$-cocycles the results from [122] in the commutative case. As in [122] the globalization accours in two steps. First we show that given a unital partial $G$-module structure on a commutative ring $\mathcal{A}$, a partial $n$-cocycle $w$ with values in $\mathcal{A}$ is globalizable if and only if a certain extendibility property holds for $w$. The second, and more technical step, consists of establishing the extendibility property, assuming that $\mathcal{A}$ is a product of blocks.

\footnotetext{
${ }^{8}$ In the case of a partial action on a semilattice, the corresponding semidirect product construction was essentially known much earlier without using the notion of a partial action (see, in particular, [249], [256]).
} 
With respect to the uniqueness of a globalization we prove that given a globalizable unital partial action $\alpha$ of $G$ on a $\operatorname{ring} \mathcal{A}$, such that $\mathcal{A}$ is a product of blocks, any two globalizations of a partial $n$-cocycle $w$ related to $\alpha$ are cohomologous. More generally, arbitrary globalizations of cohomologous partial $n$-cocycles are also cohomologous. This gives an improvement even for the case $n=2$, as we do not require that $\alpha$ is unitally globalizable. This means that the global $n$-cocycles take values in the group of the invertible elements $\mathcal{U}(\mathcal{M}(\mathcal{B}))$ of the algebra $\mathcal{M}(\mathcal{B})$ of the multipliers of $\mathcal{B}$. One should notice that the global action of $G$ on $\mathcal{B}$ naturally extends to $\mathcal{M}(\mathcal{B})$. This allows us to establish an isomorphism between the partial cohomology group $H^{n}(G, \mathcal{A})$ and the global one $H^{n}(G, \mathcal{U}(\mathcal{M}(\mathcal{B})))$.

Product of blocks were also considered in the more recent preprint [108], in which the authors characterize partial actions of groups on a finite product of indecomposable rings, dealing also with their enveloping actions.

An interesting recent advance on the globalization problem was obtained in [105], where the concept of a partial action of a group on a small weak $\kappa$-category was defined, as well as that of the corresponding partial skew category. The latter is a small weak non-necessarily associative category, a notion which is also defined in the same paper [105]. The relation of the partial skew category with the concept of the $\kappa$-algebra of a category and that of the category of a $\kappa$-algebra (with a given set of idempotents) is discussed, as well as the asocitivity of the partial skew category. Using the notion of an ideal in a category the authors define the concept of a restriction of a global action resulting in a partial action, as well as that of a globalization. Amongst other results, a criterion is given for the existence of a globalization for a partial action $\alpha$ of a group $G$ on a small weak $\kappa$-category, under the assumption that $G$ acts globally on the objects via $\alpha$. Moreover, if a globalization exists, then it is unique up to an equivalence.

Another latest development on the globalization problem was obtained in [262], were under appropriate conditions a criteria for the existence of a metrizable globalization for a given continuous partial action of a separable metrizable group $G$ on a separable metrizable space $X$ was given. If $G$ and $X$ are both Polish spaces, then the globalization is a Polish space too. The existence of a universal globalization for continuous partial actions of a countable discrete group on Polish spaces is also discussed. This topic was further studied by the same authors in [263], where amongst other results it was proved that the enveloping space (i.e. the space under the enveloping action) of a partial action of a Polish group on a Polish space is a standard Borel space.

The globalization problem from the universal algebra point of view was investigated in [210], in which a reflector of a partial action is constructed 
in the corresponding subcategory of global actions, and the question when this reflector is a globalization is considered. In particular, the notion of a partial action of a group on a relational system is introduced and it is shown that it admits a universal globalization which is a reflector. Partial group actions on partial algebras are also defined, whose domains are assumed to be relative subalgebras, and for such partial actions a necessary and sufficient condition for the existence of a globalization is given. For partial actions on total algebras the above mentioned reflector is constructed and shown to be the universal globalization. For algebras with identities the desired reflector is also produced, but it may not be a globalization. A characterization of globalizable, in the corresponding variety, partial actions is proved, which is applied to give an example of a non-globalizable partial action by taking the variety of semigroups.

The authors relate the globalization problem in a variety of algebras to embeddings of generalized amalgams into an algebra from the variety. In the case of the variety of groups this is a well-know and highly interesting topic, which was considered for other varieties too. With any partial action $\theta$ of a group on an algebra from a variety $V$ a generalized amalgam $A$ of $V$-algebras is associated, such that $\theta$ is globalizable if and only if $A$ is embeddable into a $V$-algebra. The globalization problem is also considered in [210] for partial actions on semigroups whose domains are ideals.

In the majority of cases the concept of the globalization considered by the authors is based on the restriction process described at the beginning of this survey, with domains given by formula (1). Nevertheless, it may happen that a partial action $\alpha=\left\{\alpha_{g}: X_{g^{-1}} \rightarrow X_{g}, g \in G\right\}$, extends to a global action $\beta$ in such a way that one has only the inclusions $X_{g} \subseteq Y \cap \beta_{g}(Y)$, rather than the equalities (1). Moreover, it may be useful to extend a partial action $\alpha$ on a ring $\mathcal{A}$ to a better partial action $\alpha^{*}$ on a larger $\operatorname{ring} Q$ without assuming that $\mathcal{A}$ is an ideal in $Q$. If $\alpha^{*}$ happens to be globalizable (in the above canonical sense), then the passage from $\alpha$ to the globalization of $\alpha^{*}$ is definitely an inetesting tool. These ideas lead to weaker versions of the notion of a globalization.

Such a situation appeared first in M. Ferrero's paper [169], according to which a global action $\beta$ of a group $G$ on a ring $\mathcal{B}$ is called a weak globalization of a partial action $\alpha=\left\{\alpha_{g}: D_{g^{-1}} \rightarrow D_{g}, g \in G\right\}$ of $G$ on a ring $\mathcal{A}$ if there is a monomorphism $\varphi: \mathcal{A} \rightarrow \mathcal{B}$ of rings such that

$$
\left.\beta_{g} \circ \varphi\right|_{D_{g-1}}=\varphi \circ \alpha_{g} \text { for all } g \in G \text {. }
$$


The main result in [169] says that any partial action $\alpha$ of a group $G$ on a semiprime ring $\mathcal{A}$ possesses a weak globalization. ${ }^{9}$ The proof is a fruitful idea which was used in other articles in similar situations, the main step being an extension of $\alpha$ to a unital (and therefore globalizable) partial action $\alpha^{*}$ of $G$ on the Martindale $\operatorname{ring} \mathcal{Q}$ of right quotients of $\mathcal{A}$. Then the (canonical) globalization $(\mathcal{B}, \beta)$ of $\alpha^{*}$ gives a weak globalization of $\alpha$. Notice that one does not need to assume that $\mathcal{A}$ has 1 .

A subsequent article [102] also deals with non-necessarily unital semiprime rings, but the problem was considered from the point of view of the canonical globalization. A closure condition was imposed on the domains and a property involving multipliers was used which appeared in [136] with respect to the globalization problem on $s$-unital rings. As we mentioned already, a partial actions $\alpha=\left\{\alpha_{g}: D_{g^{-1}} \rightarrow D_{g}, g \in G\right\}$, of a group $G$ on a unital $\operatorname{ring} \mathcal{A}$ is globalizable if and only of $\alpha$ is unital, i.e. each ideal $D_{g}$ is a unital ring. Now, in the case of a left $s$-unital $\mathcal{A}$ it is necessary, but not sufficient to assume that each $D_{g}$ is a left $s$-unital ring (i.e. $\alpha$ is left $s$-unital). The criterion given in [136] says that $\alpha$ is globalizable if and only of $\alpha$ is left $s$-unital and for each $g \in G$ and $a \in \mathcal{A}$ there exists a multiplier $\gamma_{g}(a)$ of $\mathcal{A}$ such that

$$
\mathcal{A} \gamma_{g}(a) \subseteq \mathcal{A} \text { and } \quad x \gamma_{g}(a)=\alpha_{g}\left(\alpha_{g}^{-1}(x) a\right), \quad \forall x \in D_{g}
$$

Now the main result in [102] says that a partial action $\alpha$ of a group $G$ on a non-necessarily unital semiprime $\operatorname{ring} \mathcal{A}$, such that each ideal $D_{g}$ is closed, is globalizable if and only if the above condition (8) is satisfied. Factoring the ring under the global action by the prime radical the authors come to a semiprime globalization, which is shown to be unique. A certain relation with the weak globalization from [169] is discussed.

In a more recent article [51] the above globalization result from [102] was refined as follows. Let $\alpha=\left\{\alpha_{g}: D_{g^{-1}} \rightarrow D_{g}, g \in G\right\}$ be a partial action of a group $G$ on a non-necessarily unital semiprime $\operatorname{ring} \mathcal{A}$ and $(\mathcal{B}, \beta)$ be the weak globalization of $\alpha$ as above. Identifying $\mathcal{A}$ with its copy in $\mathcal{B}$, write $W=\sum_{g \in G} \beta(\mathcal{A})$, and let $\beta^{\prime}$ be the restriction of $\beta$ to $W$. Observe that $W$ is a ring and $\mathcal{A}$ is an ideal in $W$ if and only if $\mathcal{A} \beta_{g}(\mathcal{A}) \subseteq \mathcal{A}$, for all $g \in G$. Producing multipliers which satisfy $(8)$ the authors prove that $\left(W, \beta^{\prime}\right)$ is a globalization of $\alpha$, provided that each $D_{g}$ is a direct summand of $\mathcal{A}$.

It is essentially more complicated to produce a weak globalization in the twisted case, which was considered in [50] with more involved use of the quotient rings technique. Let $\alpha$ be a twisted partial action of a group $G$ on a non-necessarily unital semiprime $\operatorname{ring} \mathcal{A}$. The authors in [50] extend

\footnotetext{
${ }^{9}$ In fact, it is assumed in [169] that $\alpha$ is proper, i.e. each $D_{g}$ is non-zero, however, later in [170] M. Ferrero observed that this condition is unnecessary for the proof of this result.
} 
first $\alpha$ (together with its mupliplier valued twisting) to a unital twisted partial action $\alpha^{*}$ on the left Martindale ring of quotients $Q(\mathcal{A})$ of $\mathcal{A}$. Unfortunately, there are no known tools to guarantee that $\alpha^{*}$ is globalizable, unless $Q(\mathcal{A})$ is a product of indecomposable rings [122]. So the authors go further and extend $\alpha^{*}$ to a unital twisted partial action $\alpha^{* *}$ of $G$ on the left maximal ring of quotients $Q_{m}(Q(\mathcal{A}))$ of $Q(\mathcal{A})$. Actually, one may assume that $Q_{m}(Q(\mathcal{A}))=Q_{m}(\mathcal{A})$. Now assuming in addition that $\mathcal{A}$ is a left Goldie ring, one has that $Q_{m}(\mathcal{A})$ coincides with the classical ring of quotients of $\mathcal{A}$, which is semisimple by Goldie's Theorem. Consequently, $Q_{m}(\mathcal{A})$ is a direct product of indecomposable rings and the already mentioned fact from [122] on the globalization of twisted partial actions is applicable, resulting this way in a weak globalization for $\alpha$.

Ferrero's technique [169] was used again in [27] to extend a unital (= globalizable) partial action $\alpha$ on an $\alpha$-semiprime (unital) ring $\mathcal{A}$ to the Martindale ring $\mathcal{Q}$ of $\alpha$-quotients of $\mathcal{A}$. Denoting by $(\mathcal{B}, \beta)$ the (usual) globalization of $(\mathcal{A}, \alpha)$, and extending $\beta$ to an action $\beta^{*}$ of $G$ on the Martindale ring $Q$ of $\beta$-quotients of $\mathcal{B}$, the author studies the relations between the involved actions and rings. In particular, a criterion is given when $\beta^{*}$ is a globalization for $\alpha^{*}$.

The above mentioned paper [123] extends to the partial action setting the Galois Theory of commutative rings by S. U. Chase, D.K. Harrison and A. Rosenberg [85], including several equivalent definitions of a partial Galois extension and establishing a fundamental theorem on Galois correspondence. In [85] the authors also gave the exact sequence

$$
\begin{aligned}
0 & \rightarrow H^{1}(G, \mathcal{U}(\mathcal{A})) \rightarrow \operatorname{Pic}\left(\mathcal{A}^{G}\right) \rightarrow \operatorname{Pic}(\mathcal{A})^{G} \rightarrow H^{2}(G, \mathcal{U}(\mathcal{A})) \rightarrow B\left(\mathcal{A} / \mathcal{A}^{\alpha}\right) \rightarrow \\
& \rightarrow H^{1}(G, \operatorname{Pic}(\mathcal{A})) \rightarrow H^{3}(G, \mathcal{U}(\mathcal{A})),
\end{aligned}
$$

which generalizes the two most fundamental facts from Galois cohomology of fields, namely, the Hilbert's Theorem 90 and the isomorphism of the relative Brauer group $B\left(\mathcal{A} / \mathcal{A}^{\alpha}\right)$ with the second cohomology group $H^{2}\left(G, \mathcal{A}^{*}\right)$. The latter isomorphism is obtained by associating to any 2-cocycle from $Z^{2}\left(G, \mathcal{A}^{*}\right)$ the corresponding crossed product $\mathcal{A} * G$. The above sequence was derived in [85] from the Amitsur cohomology seven terms exact sequence by S. U. Chase and A. Rosenberg [84], specifying it to the case of a Galois extension. The proof in [84] used spectral sequences and was not constructive. The first constructive proof was given by T. Kanzaki [203], introducing and applying generalized crossed products. Since then much attention have been payed to the sequence and its parts establishing generalizations and analogues in various contexts. 
Having developed the partial Galois theory in [120] on one hand, and the partial group cohomology in [124] on the other, it was reasonable to establish the analogue of the Chase-Harrison-Rosenberg exact sequence in the context of a partial Galois extension of commutative rings. The treatment in the partial action setting turned out to be more laborious, and some conceptual adjustments were needed to be made. The corresponding homomorphisms were constructed in [133], whereas the exactness of the sequence is proved in [134]. Amongst the new ingredients we introduce the Picard monoids $\operatorname{PicS}_{\mathcal{A}^{\alpha}}(\mathcal{A})$ and $\operatorname{PicS}(\mathcal{A})$, the latter being an inverse semigroup which is a disjoint union of the Picard groups of all direct summands of $\mathcal{A}$. Moreover, a partial action $\alpha^{*}$ of the Galois group $G$ on $\operatorname{PicS}(\mathcal{A})$ is used, as well as a partial action version of the generalized crossed products and two partial representations of the form $G \rightarrow \operatorname{PicS}_{\mathcal{A}^{\alpha}}(\mathcal{A})$. As the final result we have obtained the following seven-terms exact sequence

$$
\begin{aligned}
0 & \rightarrow H^{1}(G, \alpha, \mathcal{A}) \rightarrow \operatorname{Pic}\left(\mathcal{A}^{\alpha}\right) \rightarrow \operatorname{PicS}(\mathcal{A})^{\alpha^{*}} \cap \operatorname{Pic}(\mathcal{A}) \rightarrow H^{2}(G, \alpha, \mathcal{A}) \rightarrow \\
& \rightarrow B\left(\mathcal{A} / \mathcal{A}^{\alpha}\right) \rightarrow H^{1}\left(G, \alpha^{*}, \operatorname{PicS}(\mathcal{A})\right) \rightarrow H^{3}(G, \alpha, \mathcal{A}) .
\end{aligned}
$$

Other recent Galois theoretic results were produced for partial group actions on rings in [201], [202], [214], [215] and [216], and for partial coactions on coalgebras in [81]. The latest survey by A. Paques [251] gives an overview of Galois theories, inlcuding those based on partial actions.

Partial representations are in the origin of the successful approach to the study of $C^{*}$-algebras generated by partial isometries via partial actions. By a partial isomtery in a $*$-algebra $\mathcal{A}$ (in particular, in a $C^{*}$-algebra) one means an element $s \in \mathcal{A}$ with $s s^{*} s=s$. By a projection in $\mathcal{A}$ one understands a $*$-symmetric idempotent, i.e. an element $p \in \mathcal{A}$ such that $p^{*}=p$ and $p^{2}=p$. A partial *-representation $u: G \rightarrow \mathcal{A}, g \mapsto u_{g},(g \in G)$, by definition is a partial representation such that $u_{g^{-1}}=u_{g}^{*}$, for all $g \in G$. It follows from the definition that each $u_{g},(g \in G)$ is partial isometry.

A product of partial isometries is not necessarily a partial isometry, and an algebra generated by partial isometries, in general, may be rather wild, unlikely to yield to any attempt at understanding its structure. Nevertheless, algebras (abstract or $C^{*}$ ) generated by the range of a partial representation have a chance to be endowed with the structure of a crossed product by a partial action, permitting one to understand their algebraic behavior. One of the first prominent examples of the use of this technique was established in the case of the Cuntz-Krieger algebras $\mathcal{O}_{A}$ defined as follows [113]: given an $n \times n$ matrix $A=\left\{a_{i j}\right\}_{1 \leq i, j \leq n}$ with entries in $\{0,1\}$ one defines $\mathcal{O}_{A}$ as being the universal $C^{*}$-algebra generated by partial isometries $S_{1}, \ldots, S_{n}$ subject to the conditions: 
$\left.\mathrm{CK}_{1}\right) \sum_{i=1}^{n} S_{i} S_{i}^{*}=1$, and
$\left.\mathrm{CK}_{2}\right) S_{i}^{*} S_{i}=\sum_{j=1}^{n} a_{i, j} S_{j} S_{j}^{*}$.

It was shown for them in [150] that there exists a partial representation of the free group $\mathbb{F}_{n}$ sending the $i^{t h}$ canonical generator of $\mathbb{F}_{n}$ to $S_{i}$. This idea was subsequently generalized in [157] to treat the case of infinite matrices and was used to give the first definition of an analogue of Cuntz-Krieger algebras for transition matrices on infinitely many states, dropping the rowfinite condition used in earlier investigations. The algebras defined in [157] are called the Exel-Laca algebras.

The key idea is as follows. Let $u: G \rightarrow \mathcal{B}$ be a partial representation into an algebra $\mathcal{B}$. If $\mathcal{B}$ is a $*$-algebra, then $u$ is assumed to be a partial $*$ representation. It is a well-known to the experts fact that the $e_{g}=u_{g} u_{g^{-1}}$, $(g \in G)$, form a commutative set $E$ of idempotents, which are projections in the $*$-case. Let $\mathcal{A}$ be the subalgebra of $\mathcal{B}$ generated by $E$. In the $C^{*}$-case $\mathcal{A}$ is the $C^{*}$-subalgebra geberated by $E$, i.e. the smallest $C^{*}$-subalgebra of $\mathcal{A}$ containing $E$. Let $D_{g}$ be the ideal in $\mathcal{A}$ generated by $e_{g}$, i.e. $D_{g}=\mathcal{A} e_{g}$, and let $\tau_{g}: D_{g^{-1}} \rightarrow D_{g}$ be the map defined by

$$
\tau_{g}(a)=u_{g} a u_{g^{-1}}, \quad \forall g \in G .
$$

Then $\tau=\left\{\tau_{g}: D_{g^{-1}} \rightarrow D_{g}, g \in G\right\}$ is a partial action of $G$ on $\mathcal{A}$ [117, Lemma 6.5] (in the $C^{*}$-case $\tau$ is a $C^{*}$-algebraic partial action, which can be seen by carrying over the purely algebraic proof). Furthermore, in the ring theoretic case by [117, Proposition 6.8] there is a homomorphism from the crossed product (skew group ring) $\mathcal{A} \rtimes_{\tau} G$ to $\mathcal{B}$, which is an epimorphism in our case, as $\mathcal{B}$ is generated by the elements $u_{g}, g \in G$. In the $C^{*}$ case, thanks to [155, Proposition 11.14], there is a $*$-homomorphism from the (full) $C^{*}$-algebraic crossed product $\mathcal{A} \rtimes_{\tau}^{\text {full }} G$ onto $\mathcal{B}$. In some cases one is able to prove that the epimorphism obtained this way is, in fact, an isomorphism. Here we temporarily use the non-standard notation $\mathcal{A} \rtimes_{\tau}^{\text {full }} G$ for the (full) $C^{*}$-algebraic crossed product in order to make difference with the ring-theoretic crossed product $\mathcal{A} \rtimes_{\tau} G$. One should also note that in the theory of $C^{*}$-algebras there is also the so-called reduced $C^{*}$-algebraic crossed product $\mathcal{A} \rtimes_{\tau}^{\text {red }} G$ by a partial action (see [155] for details).

A very recent use of this technique in [118] made it possible to endow the Carlsen-Matsumoto $C^{*}$-algebra $\mathcal{O}_{X}$ of an arbitrary subshift $X$ with the structure of a $C^{*}$-crossed product (in this case the full and the reduced $C^{*}$ algebraic crossed products coincide). The approach based on partial actions and partial representations results in an alternative definition of $\mathcal{O}_{X}$, which 
is more convenient for our technique.

In order to describe briefly the idea of this application, let $\Lambda$ be a finite alphabet and $\Lambda^{\mathbb{N}}$ be the set of all infinite words $x_{1} x_{2} x_{3} \ldots$ in alphabet $\Lambda$ (i.e. $x_{i} \in \Lambda$ ). Taking the discrete topology on $\Lambda$ and the product topology on $\Lambda^{\mathbb{N}}$, one has that $\Lambda^{\mathbb{N}}$ is a Hausdorff compact totally disconnected space. Then the (left) shift map

$$
S: \Lambda^{\mathbb{N}} \rightarrow \Lambda^{\mathbb{N}}
$$

is defined by removing the first letter, and it is easily seen to be continuous. By a (left) subshift $X$ one means a closed $S$-invariant subset of $\Lambda^{\mathbb{N}}$. Important examples of subshifts are obtained as follows. Given an arbitrary subset $\mathcal{F}$ of finite words in alphabet $\Lambda$, called the set of forbidden words, denote by

$$
X=X_{\mathcal{F}}
$$

the set of all infinite words $x$ such that no member of $\mathcal{F}$ occurs in $x$ as an interval (contiguous block of letters). Then it is well known that $X_{\mathcal{F}}$ is a subshift and any subshift is of this form. If $\mathcal{F}$ is finite, then $X_{\mathcal{F}}$ is called a subshift of finite type. Subshifts are the objects of study of Symbolic Dynamics (see [228]), and it is interesting to bring new point of view relating algebras to them.

It turns out that there is a natural partial action on $X$, which is defined as follows. For each $a \in \Lambda$ denote by $X_{a}$ the set of all words in $X$ which begin with $a$. Restricting the shift map $S$ we obtain the function $X_{a} \rightarrow X$, whose image will be denoted by $X_{a^{-1}}$. Evidently, $X_{a^{-1}}$ consists of all $x \in X$ for which $a x \in X$. Thus the restriction of $S$ results in the partial bijection $X_{a} \rightarrow X_{a^{-1}}$, which will be denoted by $\theta_{a^{-1}}$. We also write $\theta_{a}: X_{a^{-1}} \rightarrow X_{a}$ for its inverse.

Let now $\mathbb{F}=\mathbb{F}(\Lambda)$ be the free group over $\Lambda$, i.e. the free group whose set of canonical generators is $\Lambda$. Thus with each generator $a \in \Lambda$ of $\mathbb{F}_{n}$ we may associate a partial bijection $\theta_{a}: X_{a^{-1}} \rightarrow X_{a}$ of $X$. Composing the $\theta_{a}$ 's and their inverses one may associate to each $g \in \mathbb{F}_{n}$ a partial bijection $\theta_{g}: X_{g^{-1}} \rightarrow X_{g}$ of $X$, so that $\theta=\left\{\theta_{g}, g \in G\right\}$ will be a partial action of $\mathbb{F}_{n}$ on $X$ (see $[155$, Proposition 4.10]). We call $\theta$ the standard partial action associated to $X$. In particular, any finite word $\gamma$ in alphabet $\Lambda$ is an element in $\mathbb{F}_{n}$ and

$$
\theta_{\gamma}(x)=\gamma x, \quad \theta_{\gamma^{-1}}(\gamma x)=x \quad \forall x \in X_{\gamma^{-1}} .
$$

Now taking the relative topology on $X$ one may wonder whether or not $\theta$ is a topological partial action. It follows by [118, Proposition 2.5] that $\theta$ is topological if and only if $X$ is of finite type. So we clearly have a difficulty with $\theta$ in the non-finite type case. Subshifts of finite type can be recoded and seen as Markov subshifts, i.e. subshifts obtained from graphs (see [228]). 
Since the $C^{*}$-algebras related to Markov subshifts are the Cuntz-Krieger algebras, which are well-understood from the point of view of partial actions, we are mainly interested in the case of subshifts of non-finite type, so the above mentioned problem with $\theta$ should be resolved somehow.

Despite the bad topological behavior of the standard partial action, we may use it to define a partial *-representation of $\mathbb{F}$ by bounded operators, which will be crucial for our approach. For each $g$ in $\mathbb{F}$, denote by $u_{g}$ the unique bounded linear operator

$$
u_{g}: \ell^{2}(X) \rightarrow \ell^{2}(X)
$$

such that for each $x$ in $X$,

$$
u_{g}\left(\delta_{x}\right)= \begin{cases}\delta_{\theta_{g}(x)}, & \text { if } x \in X_{g^{-1}} \\ 0, & \text { otherwise }\end{cases}
$$

where $\theta$ is the standard partial action associated to $X$. Then the map $g \mapsto u_{g}$ is a partial $*$-representation of $\mathbb{F}$ on $\ell^{2}(X)$.

Denote by $\mathcal{M}_{X}$ the closed $*$-algebra of bounded operators on $\ell^{2}(X)$ generated by $\left\{u_{g}: g \in \mathbb{F}\right\}$. It follows by [118, Proposition 6.1] that $\mathcal{M}_{X}$ coincides with the $C^{*}$-algebra defined by K. Matsumoto in [231, Lemma 4.1] (see also [75]), and we call $\mathcal{M}_{X}$ the Matsumoto algebra associated to $X$.

Denoting by $\lambda$ the left regular representation of the free group $\mathbb{F}=\mathbb{F}(\Lambda)$ on $\ell^{2}(\mathbb{F})$ and by $\left\{\delta_{g}\right\}_{g \in \mathbb{F}}$ the canonical orthonormal basis of $\ell^{2}(\mathbb{F})$, one has that

$$
\lambda_{g}\left(\delta_{h}\right)=\delta_{g h}, \forall g, h \in \mathbb{F} .
$$

Using the partial representation $u$ we define a new partial representation $\tilde{u}$ of $\mathbb{F}$ on $\ell^{2}(X) \otimes \ell^{2}(\mathbb{F})$, by tensoring $u$ with $\lambda$, namely

$$
\tilde{u}_{g}=u_{g} \otimes \lambda_{g}, \forall g \in \mathbb{F} .
$$

Then we define the Carlsen-Matsumoto $C^{*}$-algebra $\mathcal{O}_{X}$ associated to a given subshift $X$ as the closed $*$-algebra of operators on $\ell^{2}(X) \otimes \ell^{2}(\mathbb{F})$ generated by the set $\tilde{u}(\mathbb{F})=\left\{\tilde{u}_{g} g \in \mathbb{F}\right\}$. It follows from [118, Theorem 10.2] that the above defined $C^{*}$-algebra $\mathcal{O}_{X}$ is isomorphic the $C^{*}$-algebra defined by T. M. Carlsen in [73, Definition 5.1].

Having at hand the partial representation $\tilde{u}: \mathbb{F} \rightarrow \mathcal{O}_{X}$ we use the above mentioned technique (9) to produce a $C^{*}$-algebraic partial action $\tau$ of $\mathbb{F}$ on the commutative $C^{*}$-subalgebra $\mathcal{D}_{X}$ of $\mathcal{O}_{X}$ generated by the projections $\tilde{u}_{g} \tilde{u}_{g}^{*}, g \in \mathbb{F}$. One readily identifies $\mathcal{D}_{X}$ with the $C^{*}$-subalgebra of $\mathcal{M}_{X}$ generated by the projections $e_{g}=\tilde{u}_{g} \tilde{u}_{g}^{*}, g \in \mathbb{F}$, as $\tilde{u}_{g} \tilde{u}_{g}^{*}=e_{g} \otimes 1$. Then it is straightforward to shows that there exists a $*$-homorphism $\mathcal{D}_{X} \rtimes_{\tau}^{\text {full }} \mathbb{F} \rightarrow \mathcal{O}_{X}$ [118, Proposition 9.2], which is surjective, as $\mathcal{O}_{X}$ is generated by the elements 
$\tilde{u}_{g}, g \in \mathbb{F}$. In fact, further considerations and known results lead to the following isomorphisms of $C^{*}$-algebras:

$$
\mathcal{D}_{X} \rtimes_{\tau}^{\text {full }} \mathbb{F} \cong \mathcal{O}_{X} \cong \mathcal{D}_{X} \rtimes_{\tau}^{\text {red }} \mathbb{F}
$$

By Gelfand's Theorem, the $C^{*}$-algebra $\mathcal{D}_{X}$ is isomorphic to $C\left(\Omega_{X}\right)$, where $\Omega_{X}$ stands for the spectrum of $\mathcal{D}_{X}$. It is known by the $C^{*}$-theory of partial actions (see [Corollary 11.6][155]) that there is a topological partial action $\vartheta$ of $\mathbb{F}$ on $\Omega_{X}$ which corresponds to $\tau$, and which we call the spectral partial action. Using $C^{*}$-theoretic notation the above isomorphisms may be rewritten in the form

$$
C\left(\Omega_{X}\right) \rtimes_{\vartheta}^{\text {full }} \mathbb{F} \cong \mathcal{O}_{X} \cong C\left(\Omega_{X}\right) \rtimes_{\vartheta}^{\text {red }} \mathbb{F}
$$

Due to the above mentioned difficulty with the standard partial action, the main topological partial dynamical system related to an arbitrary subshift is $(\Omega, \vartheta)$, and for this reason a special attention should be payed to the topological space $\Omega$. In [118] some properties of the elements of $\Omega$ are given, so that if we look at them as subsets of the Cayley graph of $\mathbb{F}$, then they have the aspect of a river basin. It does not seem to be possible to find a complete set of properties characterizing $\Omega$, but we know that it contains a dense copy of $X$ (not necessarily with the same topology), permitting to deal with the other, more elusive elements. We are able to give necessary and sufficient conditions for such relevant properties of $\vartheta$ as minimality and topological freeness, in a rather "graphical" language, similar to those known for the case of Markov subshifts [150, Theorems 12.6, 13.19]. Then we use these conditions to give a criterion for the simplicity of $\mathcal{O}_{X}$ in terms of $X[117$, Theorem 14.5].

The above shows the importance of a partial representation $u: G \rightarrow \mathcal{A}$ when the algebra $\mathcal{A}$ (abstract or $C^{*}$ ) is generated by the range $U$ of $u$. If $\mathcal{A}$ is a $*$-algebra then in this case we say that $\mathcal{A}$ is generated by a tame set of partial isometries. Equivalently, each element of the multiplicative semigroup $\left\langle U \cup U^{*}\right\rangle$ generated by $U \cup U^{*}$ is a partial isometry (see [155, Definition 12.9 and Proposition 12.13]). Even if a $*$-algebra is generated by a wild (non-tame) set of partial isometries, one may impose additional relations on the generators in order to force them to behave well. This is concretely done in the recent paper [25] for the case of the Leavitt $C^{*}$-algebra $L_{m, n}$. The latter is the topological analogue of the algebra $L(m, n)$ considered by W. G. Leavitt in [221]. The case $L(1, n)$ is a part of the well developed theory of the Leavitt-path algebras, which are ring-theoretic analogues of the graph $C^{*}$-algebras. The generating set $U$ of $L_{m, n}$ is a wild set of partial isometries, and a way to turn around of this difficulty is to consider the $C^{*}$-algebra $\mathcal{O}_{m, n}$ which is the quotient of $L_{m, n}$ by the closed ideal generated by the elements $x-x x^{*} x$, where $x$ runs over the semigroup $\left\langle U \cup U^{*}\right\rangle$. 
This guarantees the tameness of the generating set of $\mathcal{O}_{m, n}$, allowing one to apply the above mentioned technique based on partial representations and partial actions. As a consequence, $\mathcal{O}_{m, n}$ is shown to have a full $C^{*}$-algebraic crossed product structure by a partial action (see $[25,(2.5)]$ ). It is interesting to notice that, unlike the case of algebras related to subshifts, $\mathcal{O}_{m, n}$ is not isomorphic to the corresponding reduced $C^{*}$-algebraic crossed product [25, Theorem 7.2].

Analogously to the case of (usual) representations, there is an algebra responsible for the partial representations. In the case of the $*$-representations of a group by bounded operators, the partial group $C^{*}$-algebra $C_{p}^{*}(G)$ defined in [151] plays this role, whereas its ring theoretic version $\kappa_{\text {par }} G$ is the semigroup algebra $\kappa \mathcal{S}(G)$, considered in [120]. According to a structural result from [120], if $G$ is a finite group and $\kappa$ is a commutative ring (which is assumed to be associative and unital), the the partial group algebra decomposes as follows:

$$
\kappa_{\text {par }} G \cong \bigoplus_{\substack{H \leq G \\ 1 \leq m \leq[G: H]}} \frac{b_{m}(H)}{m} \mathbb{M}_{m}(\kappa H),
$$

where $\kappa H$ stands for the group algebra of $H$ over $\kappa$ and $b_{m}(H)$ denotes the number of subsets $A \subseteq G$, such that $|A|=m|H|, 1 \in A, H=$ $\{g \in G \mid g A=A\}$. A recursive formula for the coefficients $\frac{b_{m}(H)}{m}$ given in $[120,(14)]$ was corrected in $[135,(2)]$. In [89, Theorem 2.4 ] K. Choi gave an interesting formula for the coefficients $b_{m}(H)$ using the Möbius function, but one needs to be careful with Choi's notation in [89] (see [139, Remark $3.2])$.

The proof of the above structural result used the finite groupoid $\Gamma=\Gamma(G)$ associated to a finite group $G$, the elements of $\Gamma$ being the pairs $(A, g)$, where $g \in G$ and $A$ is a subset of $G$ containing the identity $1=1_{G} \in G$ and the element $g^{-1}$. The product of pairs $(A, g) \cdot(B, h)$ in $\Gamma$ is defined for pairs for which $A=h B$, in which case:

$$
(h B, g) \cdot(B, h)=(B, g h) .
$$

Then one considers the groupoid algebra $\kappa \Gamma(G)$, which is unital with

$$
1_{\kappa \Gamma(G)}=\sum_{A \ni 1_{G}}\left(A, 1_{G}\right),
$$

together with the map $\lambda_{\mathrm{p}}: G \longmapsto \kappa \Gamma(G)$ :

$$
\lambda_{\mathrm{p}}(g)=\sum_{A \ni g^{-1}}(A, g)
$$

which turns out to be a partial representation. 
It is readily seen that any partial representation $u: G \rightarrow \mathcal{A}$ into a unital algebra $\mathcal{A}$ uniquely extends to a homomorphism of algebras $\kappa_{\text {par }} G \rightarrow \mathcal{A}$ given by $[g] \mapsto u(G),(g \in G)$. Theorem 2.6 in [120] says that a similar universal property holds for $\kappa \Gamma(G)$, from which we derive that $\kappa_{\text {par }} G \cong \kappa \Gamma(G)$, provided that $G$ is finite. This identification with the groupoid algebra then easily leads to the above structural fact.

After the publication of [120] it was mentioned in private communications independently by several researchers ${ }^{10}$ familiar with inverse semigroups that the above structural result for partial group algebras can be obtained using the theory of semigroup algebras. We spelled out this in detail in [128, Remark 2.5], without claiming novelty neither for the idea nor for the proof.

It was observed by R. Exel in [151] that the complex partial group algebras $\mathbb{C}_{\text {par }} Z_{4}$ and $\mathbb{C}_{\text {par }}\left[Z_{2} \times Z_{2}\right]$ of the cyclic group $Z_{4}$ of order 4 and the Klein-four group, respectively, are not isomorphic, whereas their usual complex group algebras are isomorphic to $\mathbb{C}^{4}$. This suggested to consider the isomorphism problem for the partial group algebras:

Which properties of $G$ are determined by $\kappa_{\text {par }} G$ ?

In particular, does the isomorphism of algebras $\kappa_{\text {par }} G_{1} \cong \kappa_{\text {par }} G_{2}$ imply $G_{1} \cong G_{2}$ ?

A negative answer for the latter question was given already in [120], producing two non-isomorphic finite non-commutative groups of order 605 with isomorphic complex partial group algebras. However, it was proved in [120, Theorem 4.4] that if $G_{1}$ and $G_{2}$ are finite abelian groups and $\kappa$ is an integral domain with char $\kappa$ not dividing $\left|G_{1}\right|=\left|G_{2}\right|$, then $\kappa_{\text {par }} G_{1} \cong \kappa_{\text {par }} G_{2}$ exactly when $G_{1} \cong G_{2}$. An analogous result in the modular case (i.e. when char $\kappa$ divides $\left|G_{1}\right|$ ) was established in [135, Theorem 3.7]. The above mentioned counter-example from [120] for the isomorphism question was improved in [138] by giving counter-examples over $\mathbb{C}$ for orders $|G|=2^{5}$ and $|G|=3^{5}$, and pointing out that there are no counter-examples for $|G|<2^{5}$ and $|G|=p^{n}<3^{5}, p \neq 2$.

Notice that in the above mentioned positive results on the isomorphism question from [120] and [135] it is assumed that both given groups $G_{1}$ and $G_{2}$ are abelian. So one may wonder whether or not the partial group algebra $\kappa_{\text {par }} G$ determines the commutativity of $G$.

More specifically, suppose that $G_{1}$ and $G_{2}$ are finite groups and $G_{1}$ is abelian. Is true that

$$
\mathbb{C}_{\text {par }} G_{1} \cong \mathbb{C}_{\text {par }} G_{2} \Longrightarrow G_{2} \text { is abelian? }
$$

\footnotetext{
${ }^{10}$ Eric Jespers, Stanley Orlando Juriaans, Boris Novikov.
} 
In [138] a list of integers was obtained which form a complete list of invariants of $\kappa_{\text {par }} G$, where $G$ is a finite $p$-group and $\kappa$ is an algebraically closed field with char $\kappa \neq p$. The invariants were expressed as sums in terms of subgroups of $G$ and numbers of their irreducible $\kappa$-representations. Then they were applied to the isomorphism problem, and it was shown, in particular, that $\kappa_{\text {par }} G$ determines the commutativity of a finite $p$-group $G$ where $p$ is an odd prime.

The list of the invariants includes, of course, the order of the group, and this does not depend on the fact of $G$ being a $p$-group. Indeed, for an arbitrary finite group $G$ the isomorphism $\kappa_{\text {par }} G \cong \kappa \Gamma(G)$ implies that the dimension of $\kappa_{\text {par }} G$ is equal to

$$
\operatorname{dim}(\kappa \Gamma(G))=\sum_{k=0}^{n-1}(k+1)\left(\begin{array}{c}
n-1 \\
k
\end{array}\right)=2^{n-2}(n+1),
$$

where $|G|=n$. The right hand side of the above formula is a strictly increasing function on $n$, so that if $G_{1}$ and $G_{2}$ are finite groups such that $\kappa_{\text {par }} G_{1} \cong \kappa_{\text {par }} G_{2}$, then $\left|G_{1}\right|=\left|G_{2}\right|$.

It turns out to be much more complicated to obtain a full list of invariants for the case when $G$ is an arbitrary finite group. Nevertheless, in [139] a series of natural invariants were given, which are useful for the isomorphism question, provided that the group order is odd. We point out the following two of them:

1) The number of Sylow $p$-subgroups of $G$ (with odd $|G|$ );

2) $\left[H: H^{\prime}\right]$ where $H$ is a Sylow $p$-subgroup of $G$.

In particular, it follows that if $|G|$ is odd, then $\mathbb{C}_{\text {par }} G$ determines the commutativity of the group $G$ [139, Corollary 5.3].

The above mentioned commutativity problem is much more difficult for finite groups of even order. In particular, the following question remains open.

Open problem: Suppose that $G_{1}$ and $G_{2}$ are finite 2-groups such that $G_{1}$ is abelian and $\mathbb{C}_{\text {par }} G_{1} \cong \mathbb{C}_{\text {par }} G_{2}$. Is it true that $G_{2}$ is also abelian?

We were not able to find a counter-example for the above problem using GAP.

The invariants in [138] were obtained using a formula for $b_{m}(H)$, which involves counting in the subgroup lattice of a finite $p$-group $G$. The formula was generalized in [139, Theorem 3.1] and used to give an alternative 
proof for the above mentioned Choi's formula [89, Theorem 2.4 ]. It was also proved that if $G_{1}$ and $G_{2}$ are finite groups such that there exists an isomorphism between the subgroup lattices of $G_{1}$ and $G_{2}$ which preserves subgroup rings, then $\kappa_{\text {par }} G_{1} \cong \kappa_{\text {par }} G_{2}$, where $\kappa$ is a commutative ring [139, Theorem 4.2]. This improves an earlier result from [135] in which it was additionally assumed that the lattice isomorphism preserves subgroups conjugation. Several examples were also given in [139], including a counterexample for the isomorphism problem of partial group algebras over $\mathbb{Q}$ with $\left|G_{1}\right|=\left|G_{2}\right|=243=3^{5}$, as well as the least counter-example over $\mathbb{C}$ in the case of odd group order $\left(\left|G_{1}\right|=\left|G_{2}\right|=189=3 \cdot 7 \cdot 9\right)$.

The decomposition of partial group algebras into a product of matrix algebras over subgroup rings gives information about the structure of the matrix partial representations of the finite group $G$. If $G$ is infinite, no such structural result is known, in particular $\kappa_{\text {par }} G$ is not isomorphic to $\kappa \Gamma(G)$, as $\kappa \Gamma(G)$ is not unital. Nevertheless, it is possible to relate the finite dimensional representations of $\kappa_{\text {par }} G$ with those of $\kappa \Gamma(G)$. To this end, for each connected component $\Delta$ of $\Gamma(G)$, whose set $\mathcal{V}_{\mathcal{D}}$ of vertices is finite, one uses an analogue $\lambda_{\Delta}: G \rightarrow \kappa \Delta$, of the partial representation (10). The map $\lambda_{\Delta}$ is defined by

$$
\lambda_{\Delta}(g)=\sum_{\substack{A \in \mathcal{V}_{\mathcal{D}} \\ A \ni g^{-1}}}(A, g),
$$

and it is shown to be a partial representation too. Identifying $K \Delta$ with a matrix algebra of the form $M_{n}(\kappa H)$ for some subgroup $H$ of $G$, the map $\lambda_{\Delta}$ becomes a partial representation of the form $G \rightarrow M_{n}(\kappa H)$, called elementary. Then the main result Theorem 2.2 of [140] says that composing the elementary partial representations of $G$ with the irreducible (usual) representations of $\kappa \Delta$ (for all such $\Delta$ ), we obtain all irreducible partial representations of $G$. A similar fact holds for the indecomposable partial representations, by replacing above the term "irreducible" by "indecomposable". The proof is rather technical, and one of the steps asserts that the subalgebra of $\kappa \Delta$ generated by the elements $\lambda_{\Delta}(g), g \in G$, coincides with $\kappa \Delta$. The argument given in [140] needed a correction which was done in [128, Proposition 2.2].

The above mentioned domains $X$ of the partial projective representations of $G$ where further studied in the recent article [128], mentioned above. It was shown already in [129] that they are exactly the $\mathcal{T}$-invariant subsets of $G \times G$, where $\mathcal{T}$ is a semigroup of order 25 acting on $G \times G$. Surprisingly, the structure of $\mathcal{T}$ does not depend on $G$ and it is a disjoint union of the symmetric group $S_{3}$ and an ideal which is a completely 0-simple semigroup. The domains $X$ form a lattice $C(G)$ with respect to the set-theoretic inclusion, intersection and union, and we know from [130, Theorem 4] that in the case of of a finite group $G$ they are exactly the finite unions of elementary 
domains, i.e. domains of elementary partial representations.

The main structural pieces in $C(G)$ are the atoms (i.e. minimal domains) and the $\mathcal{T}$-orbits which are not atoms. The latter are called blocks. Corollary 3.18 from [128] establishes the uniqueness of a minimal decomposition of a domain into a union of atoms and blocks. The domains are strongly related to the ideals of the semigroup $S_{3}(G)$ [131, Proposition 5.3], so that the above fact can be translated into the uniqueness of a decomposition of any non-zero ideal in $S_{3}(G)$ into an intersection of meet-prime ideals. The main decomposition result in [128] is Theorem 4.3 which in the case of a finite $G$ gives a decomposition of a non-minimal elementary domain into blocks and asserts its uniqueness under the assumption of minimality. On the other hand, Corollary 4.8 in [128] states the corresponding fact for the ideals in $S_{3}(G)$.

The concept of a partial group representation was extended to that of a partial representations of a Hopf algebra in [18] and [22]. In fact, the first version of a definition of a partial representation of a Hopf algebra was given in the article [18], where the globalization problem for partial Hopf actions on algebras was also studied. Influenced by [72] the latter was considered with respect to restrictions of global actions on right ideals (contrary to the above mentioned idea of restrictions on two-sided ideals). This generality resulted in an economical but an asymmetric concept of a partial representation [18, Definition 6]. In many important results on partial Hopf actions one assumes that the partial actions are symmetric $[22,(\mathrm{PA} 4)]$, a condition which is always satisfied by partial group actions. Using symmetric partial Hopf actions one comes to a new concept of a partial representation of a Hopf algebra [22, Definition 3.1] which can be obtained from the initial definition given in [18] by adding three more axioms.

An interaction between partial actions and partial representations, similar to that in [117] was also established in the Hopf case [22]. Furthermore, in [22] for any Hopf algebra $H$ a Hopf analogue $H_{\text {par }}$ of $\kappa_{\text {par }} G$ was also introduced and studied. In particular, the map $H \ni h \rightarrow[h] \in H_{\text {par }}$, where the $[h]$ 's are the defining generators of $H_{\text {par }}$ (see [22, Definition 4.1]), is a partial representation, which factors any other partial representation of the Hopf algebra $H$ [22, Theorem 4.2], similarly to the group case. The above mentioned technique (9) to produce partial crossed products from partial group representations permits one to endow $\kappa_{\text {par }} G$ with a crossed product structure $\mathcal{A} \rtimes_{\tau} G$, where $\tau$ is a partial action of $G$ on the subalgebra $\mathcal{A} \subseteq \kappa_{\text {par }} G$, generated by the idempotents $[g]\left[g^{-1}\right],(g \in G)[117$, Theorem 6.9]. Analogously, $H_{\text {par }}$ is a partial smash product of the form $\mathcal{A} \# H$ [22, Theorem 4.8], where $\mathcal{A}$ is the subalgebra of $H_{\text {par }}$ generated by the elements $\sum_{(h)}\left[h_{1}\right]\left[S\left(h_{2}\right)\right],(h \in H)$. A universal characterization of $\mathcal{A}$ is given in [22, 
Theorem 4.12] under the assumption that the antipode of $H$ is invertible.

The Hopf case turns out to be more complex, enjoying features which were invisible in the group case. In fact, $H_{\text {par }}$ has the structure of a Hopf algebroid [22, Theorem 4.10], resulting in a functor from the category of Hopf algebras to that of Hopf algebroids [22, Proposition 4.11]. Moreover, we know that if $G$ is a finite group then $\kappa_{\text {par }} G$ is finite dimensional. In the Hopf case, however, Example 4.14 shows that $H_{\text {par }}$ may be infinite dimensional, even if $H$ is finite dimensional (this is the case of the Sweedler Hopf algebra $H_{4}$ ).

The obtained facts lead to an isomorphism of the category of $H_{p a r^{-}}$ modules and the category of the so-called partial $\mathrm{H}$-modules (and naturally defined morphisms). Here the reader should notice a difference between the use of the term "partial module" in [124], [125], [126] and in [22]. As it was explained above, in the partial cohomology theory of groups by a partial $G$ module we understand a commutative monoid $A$ with a partial action of $G$ on $A$, whereas in [22, Definition 5.1], for any Hopf algebra $H$ over a field $\kappa$, by a partial $H$-module the authors mean a $\kappa$-vector space $M$ together with a partial representation of the form $H \rightarrow \operatorname{End}_{\kappa}(M)$. The latter extends to the Hopf case the notion of a $G$-space given in [140], and can be reformulated in terms of certain partial actions of $G$ on $M$ [45, Example 5.4]. Another categorical isomorphism is established between the category of symmetric partial $H$-module algebras and the algebra objects in the category of partial $H$-modules [22, Theorem 5.6].

The authors in [22] also obtained several fact on the so-called partial $G$-gradings. It is well-known that $G$-gradings on algebras are exactly the coaction of the group Hopf algebra $\kappa G$ on algebras. Thus one may define a partial grading of $G$ on an algebra $\mathcal{A}$ as a partial coaction of $\kappa G$ on $\mathcal{A}$. In particular, partial representations are applied to obtain a complete characterization of the partial $\mathbb{Z}_{2}$-gradings on algebras [22, Theorem 6.8], which, in other words, gives a transparent description of all partial superalgebras as a product of an arbitrary (usual) superalgebra and its ideal. Even earlier in [17], an example was given of a partial action of the dual group algebra $(\kappa G)^{*}$ on an ideal $I$ of the group algebra $\kappa G$, which, of course, can be considered as a partial $G$-grading on $I$.

More generally, in [15] the authors introduce and study the concept of a partial action of a Hopf algebra $H$ on a $\kappa$-category, also called a partial $H$-module category, and consider group gradings on $\kappa$-categories as a special case of Hopf module categories. Partial $H$-module categories generalize both partial Hopf actions on algebras and $H$-module categories. The latter were introduced in [94] in order to relate smash extensions to Galois coverings of $\kappa$-categories. The authors of [15] prove that any partial $H$-module category 
$\mathcal{C}$ possesses a unique, up to isomorphisms, minimal globalizatrion $\mathcal{D}$, define the notion of the partial smash product category $\mathcal{C} \# H$ and prove that $\mathcal{C} \# H$ and $\mathcal{D} \# H$ are Morita equivalent (in the sense of [ $\overline{94]}]$ ). These facts generalize the analogous results obtained earlier in [18] for Hopf actions on algebras. Furthermore, a structural result is established for the partial gradings of $\kappa$-linear categories by finite groups whose order is not divisible by char $\kappa$, and several examples are elaborated [15].

The symmetry between the algebra and coalgebra structures of a Hopf algebra, as well as the concept of the finite dual of a Hopf algebra, provides a natural ambient for duality phenomena. The duality between actions and coactions of locally compact groups on von Neumann algebras [236] inspired M. Cohen and S. Montgomery [96] to study duality between group actions and group coactions on algebras, which was later generalized by R.J. Blattner and S. Montgomery [59] for the Hopf algebra framework. In [230] the Cohen-Montgomery theorem was extended for partial actions of groups. Further generalizations were made in [19] for partial Hopf actions.

A comprehensive treatment of duality between partial Hopf actions and partial Hopf coactions was recently given in [48]. In particular, under certain (co)commutativity conditions a connection between partial (co)actions and Hopf algebroids was established in [48], one of the important steps being to endow the partial smash product $\mathcal{A} \# H$ with a structure of an $\mathcal{A}$-Hopf algebroid, provided that $H$ is cocommutative and $\mathcal{A}$ is commutative. Furthermore, for a commutative Hopf algebra $H$ and a right partial $H$-coaction on a commutative algebra $\mathcal{A}$ the authors construct a commutative $\mathcal{A}$-Hopf algebroid $\mathcal{A} \otimes H$, the so-called partial split Hopf algebroid. In the opposite direction, from certain commutative $\mathcal{A}$-Hopf algebroids the authors come to partial Hopf coactions. Moreover, a so-called skew-duality is established between $\mathcal{A} \# H$ and $\mathcal{A} \otimes H$, under certain conditions. In addition, considering the concept of a partial Hopf action and that of a partial Hopf coaction on coalgebras, an interaction between the corresponding $C$-rings and cosmash coproducts is also explored. Several other valuable facts are also proved and interesting examples are given, one of them based on algebraic groups. Without giving formal definitions, partial actions of affine algebraic groups were used earlier in [20] to produce concrete partial Hopf coactions and then, dualizing, a partial Hopf action. Now the authors formalize the definition and show, as expected, that there is a one-to-one correspondence between the partial actions of an affine algebraic group $G$ on an affine variety $M$ and the partial Hopf coactions of the coordinate Hopf algebra of $G$ on the coordinate ring of $M$ [48], which generalizes a similar classical fact known in the global case. 
Hopf algebroids appear also with respect to partial Hopf cohomology, which is the matter of the hot off the press preprint [47]. As it was mentioned above, partial $G$-modules in [124] mean unital partial actions of a group $G$ on a commutative monoid $A$. Assuming that $A$ is a (unital) algebra, it is possible to extend the notion of a partial cohomology group from [124] to the context of a partial action of a co-commutative Hopf algebra $H$ on a commutative algebra $A$ [47], giving a natural generalization of Sweedler's cohomology [278] to the partial action setting. In [124] the monoid $A$ is replaced by an appropriate inverse submonoid $\tilde{A}$, which does not change the cohomology. In the Hopf case $\tilde{A}$ is a certain quotient algebra of the symmetric algebra generated by the images of all reduced partial $n$-cochains. The algebra $\tilde{A}$ possesses a partial action of $H$, so that, as in the group case, the isomorphism $H_{p a r}^{n}(H, A) \cong H_{p a r}^{n}(H, \tilde{A})$ of the corresponding cohomology groups holds. Furthermore, $\tilde{A}$ enjoys a structure of a commutative and co-commutative Hopf algebra [47, Theorem 4.5]. In addition, by a result from [20] one naturally concludes that the partial crossed products $\underline{A \# \omega H}$ (with commutative $A$ and co-commutative $H$ ) are in a bijective cor-

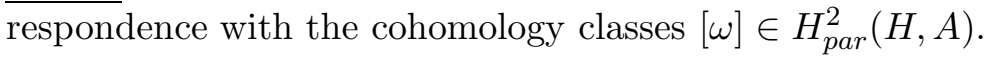

A significant new feature in [47] is the fact that the partial crossed product $\tilde{A} \#_{\omega} H$ is a Hopf algebroid over the base algebra $E(A)=\left\langle h \cdot 1_{A} \mid h \in H\right\rangle$ $[47, \overline{T h e o r e m ~ 5.10]}$. The latter suggests that it should be possible to look at the partial Hopf cohomology from the point of view of the cohomology of Hopf algebroids (see [64]). Another important Hopf algebroid comes into the picture with respect to the notion of a partially Cleft extension from [20], which, in the present setting, is shown to be a cleft extension by a Hopf algebroid in the sense of [63]. More precisely, given a co-commutative Hopf algebra $H$ acting partially on a commutative and co-commutative Hopf algebra $\tilde{A}$, and a partial 2-cocycle $\omega \in Z_{\text {par }}^{2}(H, \tilde{A})$, the partial smash product $E(\tilde{A}) \# H$ is a Hopf algebroid over $E(\tilde{A})$ by [48, Theorem 3.5]. Then [47, Theorem 6.6] states that the partial crossed product $\underline{A \# \omega H}$ is a right $E(\tilde{A}) \# H$-module algebra whose subalgebra of the co-invariants is $\tilde{A}$, and the $\overline{\text { extension }} \tilde{A} \subseteq \underline{A \#_{\omega} H}$ is a cleft extension by the Hopf algebroid $E(\tilde{A}) \# H$. Recalling from [20] that any partially cleft $H$-extension over the co-invariants can be seen as a crossed product extension, we obtain the desired fact. The article is enriched with examples and suggestions for further research.

As we mentioned already, the term "partial $G$-module", where $G$ is a group, is being understood in two related but different senses: initially the concept of a partial $G$-space was considered in [140], which means a $\kappa_{\text {par }} G$ module, or equivalently, a $\kappa$-module $M$ equipped with a partial representation $G \rightarrow \operatorname{End}_{\kappa}(M)$, and which was generalized to Hopf case in [22] to deal with partial Hopf representations; the other one means a partial action of 
$G$ on a commutative semigroup (whose domains are assumed to be ideals), and is used to deal with partial cohomology in [47], [124], [125], [126], [127], [133], [134], [137] and [206]. Of course, it is also natural to take the first meaning and define the corresponding cohomology of $G$, as it has been recently done in [16]. More precisely, denote by $B$ the commutative subalgebra of $\kappa_{\text {par }} G$ generated by all idempotents $e_{g}=[g]\left[g^{-1}\right], g \in G$ (this subalgebra was denoted above by $\mathcal{A}$, but we prefer now to reserve the symbol $\mathcal{A}$ for a general algebra). The partial representation $G \ni g \mapsto[g] \in \kappa_{\text {par }} G$ results by (9) in a unital partial action $\tau$ of $G$ on $B$, which in its turn gives rise to a partial representation $\lambda: G \rightarrow \operatorname{End}_{\kappa}(B)$, by the standard formula

$$
\lambda(g): B \ni b \mapsto \tau_{g}\left(b e_{g^{-1}}\right)=[g] b\left[g^{-1}\right] \in B .
$$

The latter endows $B$ with a structure of a left $\kappa_{\text {par }} G$-module, and one may consider $\kappa_{\text {par }} G$ as an augmented ring (see [76, Chapter VIII]) by means of the left $\kappa_{\text {par }} G$-module epimorphism $\varepsilon: \kappa_{\text {par }} G \rightarrow B$, defined by

$$
s=\left[g_{1}\right]\left[g_{2}\right] \ldots\left[g_{n}\right] \mapsto e_{g_{1}} e_{g_{1} g_{2}} \ldots e_{g_{1} g_{2} \ldots g_{n}}=s s^{-1} .
$$

Then one takes the cohomology of the augmented $\operatorname{ring} \kappa_{\text {par }} G \rightarrow B$ with values in an arbitrary $\kappa_{\text {par }} G$-module $M$ [16], i.e. one defines

$$
H_{p a r}^{n}(G, M)=\operatorname{Ext}_{\kappa_{\mathrm{par}} G}^{n}(B, M) .
$$

Notice the difference between this use of the notation $H_{p a r}^{n}$ and that in the above mentioned paper [47]. Observe also that the ordinary group cohomology can be seen as the cohomology of the augmented ring $\kappa G \rightarrow \kappa, g \mapsto 1$, with values in a $\kappa G$-module, whereas, the Hochschild cohomology of an associative algebra $\mathcal{A}$ can be defined as the cohomology of the augmented ring $\mathcal{A} \otimes \mathcal{A}^{o} \rightarrow \mathcal{A}, a \otimes a^{\prime} \mapsto a a^{\prime}$, with values in an $\mathcal{A} \otimes \mathcal{A}^{o}$-module (i.e. an $\mathcal{A}$ bimodule), where $\mathcal{A}^{o}$ stands for the opposite algebra of $\mathcal{A}$ (see [76]). Given a unital partial action $\alpha$ of a group $G$ on an algebra $\mathcal{A}$ over a field $\kappa$, the authors of [16] use Grothendieck's result [268, Theorem 10.47] in order to prove the existence of a spectral sequence relating the Hochschild cohomology of the partial skew group ring $\mathcal{A} \rtimes_{\alpha} G$ with $H_{p a r}^{n}(G,-)$ and the Hochschild cohomology of $\mathcal{A}$.

To compare the cohomology from [16] with that from [124], one may adapt the projective resolution given in [124] to the case of vector spaces, so that the $n$-cocycles and $n$-coboundaries are defined by similar formulas. This may give the impression that the two cohomologies coincide. Nevertheless, one should notice a significant difference in the concepts of $G$-modules. A $\kappa G$-module can be seen as a partial action of $G$ on a vector space [45, Example 5.4]. In the definition of a partial group action on a vector space (see [8, Remark 2.3]) one assumes that each domain is a subspace and the involved bijections are $\kappa$-maps. It is evident that the category of $\kappa G$-modules is abelian. On the other hand, the partial $G$-modules from [124] are partial actions of $G$ on commutative semigroups. Their domains are unital 
two-sided ideals in the semigroup. Thus, if the semigroup under the partial action turns out to be a group, then the action has to be global. It is pointed out in [124] that the category of partial $G$-modules is not abelian. The requirement that the domains are ideals is essential to fit the theory of twisted partial group actions on algebras. As a consequence, it allows one to define partial crossed products in the category of inverse semigroups and interpret low-dimensional cohomology groups in terms of extensions. Furthermore, important structural results in semigroup theory use partial actions on meat-semilattices whose domains are order ideals. Semilattices can be considered as semigroups, and order ideals are semigroup ideals.

A natural situation in which both cohomologies appear is that of a unital partial action $\alpha$ of a group $G$ on a commutative algebra $\mathcal{A}$. The domains are ideals generated by idempotents $1_{g}, g \in G$, in particular, they are vector subspaces. In this case the above formula (11) becomes

$$
\pi(g): \mathcal{A} \ni a \mapsto \alpha_{g}\left(a 1_{g^{-1}}\right) \in \mathcal{A}
$$

which gives a partial representation $\pi$ of $G$ by $\kappa$-linear transformations of $\mathcal{A}$. Thus the cohomology from [16] deals with the additive structure of $\mathcal{A}$. Focusing on the multiplicative semigroup of $\mathcal{A}$ one has a partial $G$-module in the sense of [124] and the corresponding cohomology is based on the multiplicative structure of $\mathcal{A}$. This shows that the cohomology groups from the two theories have little in common, except for the case $n=0$, in which both of them are related to partial invariants.

The importance of groupoids for partial representations was mentioned above. On the other hand, in [5] a locally compact groupoid (called transformation groupoid) was related to any continuous partial action $\theta$ of a second countable locally compact Hausdorff group $G$ on a second countable locally compact Hausdorff space $X$ and used to prove that the $C^{*}$-algebra of the groupoid is isomorphic to the $C^{*}$-crossed product $C_{0}(X) \rtimes_{\theta}^{\text {full }} G$ (here the partial action of $G$ on $C_{0}(X)$, dual to that of $G$ on $X$, is denoted by the same symbol $\theta$ ). In the discrete group case another way to associate a groupoid to a partial action was given in [6]. More recent results involving groupoids related to partial actions include the groupoid $C^{*}$-algebra characterization of the enveloping $C^{*}$-algebras associated to partial actions of countable discrete groups on locally compact spaces with applications in [156], as well as the study of the amenability of groupoids related to partial semigroup actions, englobing groupoids associated to directed graphs, higher rank graphs and topological higher rank graphs in [265]. Moreover, an algebraic version of the above mentioned result from [5] was established in [53] which says that given a continuous partial action of a discrete group $G$ on a locally compact totally disconnected Hausdorff space with clopen domains, the Steinberg algebra of the associated transformation groupoid and the skew group ring 
by the corresponding partial action of $G$ on the compactly supported locally constant function algebra are isomorphic. In the same article [53] the authors show that any Steinberg algebra ([95], [277]), associated to an ample Hausdorff groupoid, can be seen as a partial skew inverse semigroup ring.

The use of groupoids was essential in [235] for partial crossed product characterizations of certain inverse semigroup $C^{*}$-algebras. This was done by dealing with the above mentioned transformation groupoid of a partial group action from [5] (also defined independently in [205] in the discrete case), as well as with the universal groupoid $\mathcal{G}(S)$ of an inverse semigroup $S$. The latter is the groupoid of germs of a certain natural action of $S$ on the space of the semi-characters $\hat{E}$, where $E=E(S)$ stands for the idempotents of $S$. The groupoid of germs of an inverse semigroup action is defined in a similar spirit with the transformation groupoid of a partial action, taking into account the natural partial order of $S$. The reader is referred to [154] for a detailed construction. The authors of [235] prove that if $S$ is a countable $E$ unitary (or strongly 0-E-unitary) inverse semigroups, then there is a partial action $\theta$ of the maximum group image $G$ of $S$ on $\hat{E}$ such that $\mathcal{G}(S)$ is isomorphic to the transformation groupoid of $\theta$. This led to the above mentioned crossed product descriptions. Moreover, the tight spectrum $\hat{E}_{\text {tight }}$ (see [154]) is invariant under the action of $S$, and it is shown in [235] that the corresponding groupoid of germs $\mathcal{G}_{\text {tight }}(S)$ is also isomorphic to a transformation groupoid of a partial action of $G$. As a consequence, the full $C^{*}$-algebra of $\mathcal{G}_{\text {tight }}(S)$ is isomorphic to the crossed product $C_{0}\left(\hat{E}_{\text {tight }}\right) \rtimes^{\text {full }} G$. The latter furnishes a general explanation for a number of partial crossed product facts.

Apart from their importance to partial (semi)group actions and partial group representations, groupoids became an object of study also subject to their own partial actions. This was influenced by the paper [178], in which the definition of a partial action of an ordered groupoid on a set was given, and it was proved that any such partial action is globalizable. Furthermore the Birget-Rhodes expansion $\tilde{G}^{R}$ of an ordered groupoid $G$ was defined and it was shown that there is a one-to-one correspondence between the partial actions of $G$ on a set $X$ and the actions of $\tilde{G}^{R}$ on $X$, extending R. Exel's result from [151], mentioned above.

The study of partial groupoid action on rings began in the working group leaded by Antonio Paques, the first publication being [40], in which the notion of a partial action $\alpha$ of an ordered groupoid $G$ on a $\operatorname{ring} \mathcal{A}$ was introduced and the corresponding partial skew groupoid $\operatorname{ring} \mathcal{A} \rtimes_{\alpha} G$ was defined. It was also proved that the meet-preserving actions of $\tilde{G}^{R}$ on $\mathcal{A}$ are in a one-to-one correspondence with those partial actions of $G$ on $\mathcal{A}$, in which the domain of each partial bijection is an ideal in $\mathcal{A}$. Furthermore, if $\alpha$ is a partial action of $G$ on $\mathcal{A}$ and $\beta$ is the corresponding action of $\tilde{G}^{R}$ on 
$\mathcal{A}$ then $\mathcal{A} \rtimes_{\alpha} G$ is an epimorphic image of $\mathcal{A} \rtimes_{\beta} \tilde{G}^{R}$, which extends a result from [163], obtained in the case of partial group actions. Analogous facts for partial actions of inductive groupoids on algebras were obtained in [37], motivated by the fact that the category of inverse semigroup is isomorphic to that of the inductive groupoids.

The concept was further generalized in [41], where the definition of a partial action of an arbitrary groupoid on a ring was given, a criterion for its globalization was proved under the assumption that the identities of the groupoid act (trivially) on unital rings, showing also that the partial skew groupoid ring and the corresponding global one are Morita equivalent. The latter fact was used to establish Galois theoretic results. More general globalization and Morita equivalence facts were recently obtained in [42], where the unital condition on the above mentioned domains was replaced by the $s$-unital condition. This extends also the results in [136] for partial group actions on $s$-unital rings.

The general definition from [41] gives, in particular, a notion of a groupoid action, used in [174], where Cohen-Montgomery-type duality theorems for (co)actions of groupoids were proved, and in [173] to study the Azumaya property for skew groupoid rings, obtaining a nice relation with the notions of a Galois extension and Hirata-separability. Another recent development is a Galois theory in the style of Grothendieck and Dress for groupoids acting on commutative rings [254], including a Galois correspondence result. The definition of a (global) groupoid action in [41] is equivalent, under some conditions, to that given in [70]. Furthermore, in a latest article [43] necessary and sufficient conditions for the skew groupoid ring $A *_{\alpha} G$ to be a separable extension of $A$ were given, where $G$ is a finite connected groupoid acting partially on a ring $A$. For a general $G$ with finite number of objects the problem of separability is reduced to the connected case. If an arbitrary groupoid $G$ acts globally on $A$ then the separability of the extension forces $G$ to be finite.

The globalization problem in the general setting of partial category actions was investigated in the new article [244], in which the concept of a partial action of a category on a set is defined, as well as that of a continuous partial action of a topological category on a topological space. It is proved that any partial action of a category on an arbitrary set admits a universal globalization, and the latter fact is also true in the context of continuous partial category actions. It is also shown that the new concept fits nicely the notion of a partial action of a groupoid on a set.

A new advance produced by the group of A. Paques comes from the fact that groupoid algebras and Hopf algebras are both englobed by weak Hopf algebras. The partial actions of weak Hopf algebras on rings were defined in [78] and studied with respect to the globalization problem, and the existence 
of a Morita context between the partial smash product and the corresponding global one. A difficulty arises here when defining partial smash products due to the fact that the tensor product is taken not over the ground field. Amongst various interesting results a complete characterization of the partial actions of a weak Hopf algebra on the base field was given, suggesting a way to produce new examples. The authors also study the relation between partial groupoid actions, in the sense of [41], and partial actions of groupoid algebras as weak Hopf algebras, showing how partial groupoid actions fit into this general context.

A Hopf algebra $H$ may act partially on an algebra $\mathcal{A}$ from the right and from the left, and if these two actions commute, then we have the concept of a partial $H$-bimodule algebra as defined by A. Paques et al. in [79]. Similarly, the authors of [79] define partial $H$-bicomodule algebras and study the globalization problem for both structures. The relations between the partial $H^{0}$-biactions and the partial $H$-bicoactions is studied, where $H^{0}$ stands for the finite dual of $H$. In addition, under the assumption that $H^{0}$ separates points, the authors prove that the globalization of a partial $H^{0}$-bimodule algebra and that of the corresponding partial $H$-bicomodule algebra are isomorphic as $H^{0}$-bimodule algebras.

As a dual counterpart of the globalization theorems for partial Hopf (co)actions on algebras in [18] and [19], the globalization problem for partial Hopf (co)actions on coalgebras was recently considered in [80]. More precisely, it is proved that every partial module coalgebra is globalizable and, under a certain rationality condition, the globalization for a partial comodule coalgebra also exists. The relations between actions and coactions on algebras and coalgebras are analyzed, as well as between their globalizations (sometimes assuming some natural conditions). Interesting examples are also given.

Multiplier algebras entered the theory of partial actions at the stage of its foundations: R. Exel's definition of a continuous twisted partial action of a locally compact group on a $C^{*}$-algebra uses invertible multipliers of certain ideals as values of the twist [149]. This was transferred to abstract algebras [121], as well as to semigroups [126]. In purely ring theoretic setting, multipliers turned out to be crucial to deal with the associativity of partial crossed products [117], [121], whose arguments were also used for semigroups in [124], [125] and [126], and which also provided an algebraic proof of the associativity of partial $C^{*}$-crossed products, which was initially achieved by means of approximate identities. Furthermore, multipliers are essential ingredients of the crossed product criterion for graded algebras [121, Theorem 6.1], as well as of the more general partial cohomology theory [126]. 
On the other hand, multipliers came into the Hopf theoretic picture in A. Van Daele's definition of "non-unital Hopf algebras", i.e. the so-called multiplier Hopf algebras [280], in which the co-multiplication takes values in the multiplier algebra of the tensor product, $\Delta: H \rightarrow M(H \otimes H)$. This concept was born in 1994, and since then much attention is being payed to various aspects of multiplier Hopf algebras and their generalizations. In particular, A. Paques introduced the authors of the hot of print article [36] to the subject and suggested to investigate their actions and co-actions on non-necessarily unital algebras. The notion of a partial action of a regular multiplier Hopf algebra $H$ on an algebra $R$ with a nondegenerate product is introduced and studied in [36], as well as that of a coaction of $H$ on $R$. The relation between the actions and coactions is discussed. The main result gives, under natural assumptions, a Morita context connecting the coinvariant algebra $R \underline{\mathrm{coH}}$ with an appropriate smash product. Finally a notion of a partial Galois coaction is related to this Morita context.

A rather general version of Hopf Galois theory was elaborated in [14], dealing with the so-called lax entwining structures introduced by S. Caenepeel and K. Janssen in [71] ${ }^{11}$, and their particular cases of weak and partial entwining structures. Weak entwining structures can be obtained from comodule algebras over weak bialgebras, whereas partial entwining structures can be constructed from partial actions of bialgebras on algebras. Each of these entwining structures consists of an algebra $\mathcal{A}$ and a coalgebra $\mathcal{C}$ together with a map $\psi: \mathcal{C} \otimes \mathcal{A} \rightarrow \mathcal{A} \otimes \mathcal{C}$ obeying certain axioms. A classical result by Y. Doi and M. Takeuchi (1986) states that an $H$-extension is cleft precisely when it is Hopf Galois with normal basis. The authors extend this fact to the general context of lax Galois extensions in a symmetric monoidal category, by introducing lax $\mathcal{C}$-cleft extensions and proving that the lax $\mathcal{C}$-cleft extensions are exactly the lax $\mathcal{C}$-Galois extensions with normal basis. Further results on $\mathcal{C}$-Galois extensions, as well as on various types of entwining structures were obtained in [274].

An important part of the research on partial actions is dedicated to ideals and the ring theoretic properties of partial crossed products. First of all, one asks whether or not the partial crossed products are associative. It turned out that it is not always the case. In [117] a $\operatorname{ring} \mathcal{A}$ was called strongly associative if for any group $G$ and any partial action $\alpha$ of $G$ on $\mathcal{A}$, the partial skew group ring $\mathcal{A} \rtimes_{\alpha} G$ is associative. In the language of multipliers a sufficient condition for the associativity of $\mathcal{A} \rtimes_{\alpha} G$ was given in [117], in particular, semiprime rings are strongly associative. This was extended in [40] to partial skew groupoid rings. On the other hand, the ring of the uppertriangular $n \times n$-matrices over a field is strongly associative if and only if $n \leq 2$. Another example of the non-associativity was given by constructing

\footnotetext{
${ }^{11}$ The preprint [71] was a preliminary version of [72].
} 
a partial action of the group of order 2 on a four-dimensional algebra, which in the case of characteristic 2 is isomorphic to the modular group algebra of the Klein-four group. This suggested the problem of the characterization of strongly associative modular group algebras of finite groups, which was solved in [192], [193] and [194]. In the recent preprint [195] the strongly associative modular semigroup algebras $\kappa S$ are characterized, where $S$ is a finite inverse semigroup without zero, which is not a group, and $\kappa$ is an algebraically closed field whose characteristic $p$ divides the order of the kernel of $S$. As a consequence, it is pointed out that the modular partial group algebra $\kappa_{\mathrm{par}} G$ is never strongly associative, provided that $G$ is a finite group and $\kappa$ is an algebraically closed field whose characteristic divides $|G|$.

A systematic ring-theoretic investigation of partial skew group rings, the rings under partial actions and the rings under their enveloping actions began in [171], whereas the study of the prime and maximal ideals in [101] initiated the consideration of partial skew polynomial rings. Subsequent results were obtained in [106], [32], [30], [98], [104], [27], [29], [77], [99] and [103]. The treatment of the ring-theoretic properties of the more general structure of a crossed product by a twisted partial group action began in [39] and [253]. In particular, results on the Azumaya property from [253] were important to define in [133] a homomorphism from the partial cohomology group $H^{2}(G, \alpha, \mathcal{A})$ to the relative Brauer group $B\left(\mathcal{A} / \mathcal{A}^{\alpha}\right)$ by sending any arbitrary partial 2-cocycle to the corresponding crossed product. This map fits into the seven terms exact sequence [134], mentioned above, and in the case of a (global) Galois extension of fields results in the classical isomorphism between the second cohomology group and the relative Brauer group.

More recent developments on the ring theoretic properties include the study of the simplicity of the partial crossed products in [183], [184], [44], [245] and [246], and the chacarterization of the Leavitt path algebras as partial group rings [186], which permitted one to obtain alternative proofs for the simplicity criterion and for the Cuntz-Krieger uniqueness theorem for Leavitt path algebras (see [186] and [184]). Furthermore, in [35] the relations between the module properties over $\mathcal{A}, \mathcal{A} \rtimes_{\alpha} G$ and $\mathcal{A}^{\alpha}$ were studied, where $\mathcal{A}^{\alpha}$ denotes the subring of the $\alpha$-invariants, whereas in [107] the related structure of the partial skew power series ring was recently considered with respect to the distributive and Bezout duo properties.

As to more general skew structures, in [190] the Leavitt path algebras were characterized as partial skew groupoid rings, which was applied to study a class of groupoid graded isomorphisms between Leavitt path algebras. In [54] the simplicity of the skew group ring by a partial action of an inverse semigroup on a commutative ring was characterized and used to offer a new proof for the simplicity criterion for a Steinberg algebra associated with a 
Hausdorff and ample groupoid. Furthermore, in [82] the semiprimitivity and the semiprimality properties for partial smash products were considered, as well as their prime and Jacobson radicals.

In a recent preprint [247] a rather general situation of a unital partial (in particular, global) action of a groupoid $G$ on a non-necessarily associative ring $R$ was considered. In particular, it was proved that if the partial action is unital and $R$ is alternative, then $R * G$ is left (or right) artinian if and only if so too is $R$ and $R_{g}=\{0\}$ for all but finitely many $g \in \operatorname{mor}(G)$. In the global case the assumption on $R$ to be alternative can be dropped. This extends the well-known result of 1963 by I. G. Connel on group rings, as well as its more general version for global skew group rings by J. K. Park (1979). Applications were given to groupoid rings, partial group rings, generalized matrix rings and Leavitt path algebras, and, moreover, the noetherian property for partial skew groupoid rings was also considered.

For the twisted case in [50] the authors use the above discussed weak globalization for a twisted partial action $\alpha$ of a group $G$ on a semiprime ring to give several results with necessary and sufficient conditions for a partial crossed product $\mathcal{A} *_{\alpha} G$ to be a semiprime left Goldie ring, imposing reasonable restrictions, such as $G$ being finite and $\mathcal{A}$ having no $|G|$-torsion, or $G$ being an infinite polycyclic group and $\alpha$ being of finite type etc. In order to obtain these results the authors establish a series of facts relating the properties and ideals of $\mathcal{A} *_{\alpha} G$ to those of $Q_{m}(\mathcal{A}) *_{\alpha} G$. In particular, if $\mathcal{A} *_{\alpha} G$ is semiprime left Goldie ring, then so too is $\mathcal{A}$, without any restriction on $G$ and $\alpha$.

More recently, in [49] partial crossed product by twisted partial actions were considered with respect to the artinian, noetherian, semilocal, perfect and semiprimary properties, under some assumptions on the twisted partial actions, such as to be of finite type. If the twisted partial action is globalizable, then the above ring theoretic properties for the partial crossed products were related to those for the corresponding global crossed product. Moreover, the Krull dimension of partial crossed products was also studied in [49], as well as the global dimension and the weak global dimension. Furthermore, triangular matrix representations of partial skew group rings were also considered, and, in addition, it was shown with certain assumptions that the ring under the twisted partial action is Frobenius or symmetric, then so too is the crossed product.

In another preprint [109] twisted partial skew power series rings and twisted partial skew Laurent series rings are introduced and studied with respect to the properties of being a prime ring, a semi-prime ring, a semiprime Goldie ring or a Goldie ring with semiprime base subring. Prime ideals are considered as well as the prime radical (the latter for the twisted partial 
skew power series case).

In relation to the twisted case in [248] the authors introduce the concept of a unital ring $S$ which is epsilon strongly graded by a group $G$, as a simultaneous generalization of the strongly graded rings and crossed products by unital twisted partial actions. They define a trace map and using it give a criterion of the separability of the extension $S_{1} \subseteq S$. This generalizes a known result for strongly graded rings, as well as the corresponding result from the above mentioned paper [39]. Amongst other facts, the hereditary and Frobenius properties, and the simplicity for epsilon-strongly graded rings are also discussed, and the so-called epsilon-crossed products are introduced, which are proved to be isomorphic with the unital partial crossed products. A series of examples are worked out, including those giving separable epsilonstrongly graded rings, which are neither strongly graded, nor partial crossed products in some natural way.

As to the Hopf case, in [83] a general theory of partial $H$-radicals is developed for partial $H$-module algebras based on the Amitsur-Kurosh general radical theory. Using the monoidal category of left partial $H$-modules from [22] and its subcategory of algebra objects, a definition of a partial smash product $A \# H$ is elaborated, where $H$ is a Hopf algebra with a bijective antipode and $A$ is a non-necessarily unital $H$-module algebra (in the above mentioned subcategory). Several examples of partial $H$-radicals of Jacobson type are worked out and studied. The obtained results extend facts established in the global case in [172] and [270]. Some questions posed in [172] are also discussed.

In [282] for a finite dimensional Hopf algebra $H$ over a field, imposing a condition of a lazy 1-cocycle [55], the concept of a strong partial $H$-module algebra is defined. For strong partial $H$-module algebras the partial trace map is considered and a Maschke-type theorem is proved. Separability of the partial smash product over the base ring is also discussed.

The partial trace map naturally occurred in earlier papers, and it is related to both Galois theory and Morita theory. Amongst the above mentioned Morita theoretic facts, two type of Morita equivalence results are used in the algebraic investigations of ring theoretic properties. The first one has its origin in [4], where it was proved that if $\alpha$ is a globalizable partial action of a locally compact Hausdorff group $G$ on a $C^{*}$-algebra $\mathcal{A}$ and $(\mathcal{B}, \beta)$ is its enveloping action, then the reduced $C^{*}$-crossed products $\mathcal{A} \rtimes_{\alpha}^{\text {red }} G$ and $\mathcal{A} \rtimes_{\beta}^{\text {red }} G$ are strongly Morita equivalent. We already referred to the recent $C^{*}$-theoretic extensions of this fact in [7] and [10]. Algebraic versions appeared in [117], [136], [121], [18], [41], [15], [78], [42]. The other type of results is inspired by the global case and for partial group actions appeared 
in [32]: given a unital partial action of a group $G$ on a ring $\mathcal{A}$, there is a natural Morita context linking the subring of the $\alpha$-fixed points $\mathcal{A}^{\alpha}$ and the skew group ring $\mathcal{A} \rtimes_{\alpha} G$; the context is strict if and only if $\mathcal{A}^{\alpha} \subseteq \mathcal{A}$ is an $\alpha$-Galois extension. Its analogue for partial Hopf actions was established in [17], and Hopf theoretic considerations about the corresponding Morita context is the topic of a recent survey by A. Paques [250]. The discussion in [250] involves the partial trace map, the strictness of the context, Galois extensions, separability, and is accompanied by a series of illustrative examples.

Considerations on ideals in partial crossed products naturally apper when dealing with ring-theoretic properties, however, they are of great interest on their own. Thus in one of the latest papers [100] the author investigate the prime Goldie ideals in the related structure of the partial skew polynomial rings, whereas in [52] the ideals are studied in skew group rings by partial actions on algebras of functions on an abstract set $X$.

More precisely, let $\kappa$ be a field and denote by $\mathcal{F}_{0}(X)$ the algebra of all functions $X \rightarrow \kappa$ with finite support, endowed with the pointwise operations. Then there is a bijection between the non-zero ideals of $\mathcal{F}_{0}(X)$ and the non-empty subsets of $X$, and, moreover, there is a one-to-one correspondence between the partial actions of a group $G$ on $X$ and the partial actions of $G$ on $\mathcal{F}_{0}(X)$. If the partial action $\alpha$ of $G$ on the set $X$ is free, then the skew group ring $\mathcal{F}_{0}(X) \rtimes_{\alpha} G$ is shown to be isomorphic to a certain equivalence relation algebra, and this is used to describe the ideals of $\mathcal{F}_{0}(X) \rtimes_{\alpha} G$ as function rings on appropriate subsets of $X$. The results are analogues of known facts on partial actions on the $C^{*}$-algebra $C_{0}(X)$, where $X$ is a locally compact Hausdorff space. As a motivation, the authors include a proof of a simplified version of the above mentioned F. Abadie's characterization of $C^{*}$-partial crossed products as groupoid $C^{*}$-algebras, for the case of free partial actions of countable groups acting on unital commutative $C^{*}$-algebras.

There is a rich history of the study of ideals in $C^{*}$-algebraic crossed products, with a special role played by the so-called Effros-Hahn conjecture [145], which in its original form states that every primitive ideal in the crossed product of a commutative $C^{*}$-algebra by a locally compact group should be induced from a primitive ideal in the $C^{*}$-algebra of some isotropy group. It was proved in [269] in the case of discrete amenable groups and later extended to various contexts.

In [119] the Effros-Hahn conjecture is studied in the algebraic setting, more specifically, for the skew group rings $\mathscr{L}_{c}(X) \rtimes G$, where $G$ is an arbitrary discrete group acting partially on a locally compact Hausdorff, totally disconnected topological space $X$, and $\mathscr{L}_{c}(X)$ stands for the algebra of all locally constant, compactly supported functions $X$ with values in an arbitrary field $\kappa$. It is also assumed that each domain $X_{g}$ in the partial action 
is closed and open (clopen) in $X$. One of the main results in [119] says that every ideal in $\mathscr{L}_{c}(X) \rtimes G$ is an intersection of ideals induced from isotropy groups.

Tools are developed in order to understand the induction process, one of them being the notion of an admissible ideal of the group algebra $\kappa H_{x_{0}}$, where $H_{x_{0}}$ is the isotropy group of a point $x_{0} \in X$. In particular, denoting by $\operatorname{Ind}_{x_{0}}(I)$ the ideal of $\mathscr{L}_{c}(X) \rtimes G$ induced from an ideal of $I$ of $\kappa H_{x_{0}}$, the map $I \mapsto \operatorname{Ind}_{x_{0}}(I)$ is proved to be an injection of the set of the admissible ideals of $\kappa H_{x_{0}}$ into the set of the ideals of $\mathscr{L}_{c}(X) \rtimes G$. In order to compare ideals induced from distinct isotropy groups the notion of a transposition of ideals is introduced, which takes any ideal of $\kappa H_{x_{0}}$ to an admissible ideal of $\kappa H_{\hat{x}_{0}}, x_{0}, \hat{x}_{0} \in X$. It is proved in [119] that given admissible ideals $I \triangleleft \kappa H_{x_{0}}$ and $\hat{I} \triangleleft \kappa H_{\hat{x}_{0}}$, the equality $\operatorname{Ind}_{x_{0}}(I)=\operatorname{Ind}_{\hat{x}_{0}}(\hat{I})$ occurs if and only if $\hat{I}$ is the transposition of $I$. A concrete description of a transposed ideal is given, and it is also shown that the induction preserves some important properties of the ideals, namely, being primitive, or prime, or meet-irreducible.

Notice that the above mentioned assumption on $X_{g}$ to be closed (besides being open by definition) naturally appears in [156]: the enveloping space of a topological partial action of a countable discrete group on a compact Hausdorff space is Hausdorff if and only if each domain $X_{g}$ is closed (note that we already mentioned that this condition was also used in [53]).

The ideas of [119] were further developed in the most recent preprint [114] to the study of the ideals in the Steinberg algebra $A_{\kappa}(\mathcal{G})$ of an ample Hausdorff groupoid $G$ over a field $\kappa$, by considering $A_{\kappa}(\mathcal{G})$ as as an inverse semigroup crossed product algebra. As in [119], it is proved that every ideal in $A_{\kappa}(\mathcal{G})$ may be obtained as the intersection of ideals induced from isotropy group algebras. Since the partial skew group $\operatorname{ring} \mathscr{L}_{c}(X) \rtimes G$ from [119] can be viewed as the Steinberg algebra [277] for the transformation groupoid associated to the partial action of $G$ on $X$, the algebraic version of the Effros-Hahn conjecture proved in [119] is extended now to the generality of Steinberg algebras.

Recent works around partial actions include also the study of the category of the partial Doi-Hopf modules in [86], of partial actions on power sets in [34], of the category of partial $G$-sets for a fixed group $G$ in [28], of partial orbits and $n$-transitivity in [31], of partial group entwining structures and partial group (co)actions of a Hopf group coalgebra on a family of algebras in [87], of generalized partial smash products in [143], and of twisted partial Hopf coactions and corresponding partial crossed coproducts in [88], as well as a note on sums of ideals [33]. More information around partial actions may be found in the surveys [45], [115], [116], [170], [250], [251] and [260]. 
Notice also that partial actions and/or related structures have been mentioned in [12], [13], [24], [46], [62], [67], [90], [92], [142], [154], [161], [167] and [166].

\section{ACKNowledgements}

The author thanks Fernando Abadie and Mykola Khrypchenko for many useful comments.

\section{REFERENCES}

1. B. Abadie, S. Eilers, R. Exel, Morita equivalence for crossed products by Hilbert C*-bimodules, Trans. Amer. Math. Soc., 350 (1998), 3043-3054.

2. B. Abadie, F. Abadie, Ideals in cross sectional $C^{*}$-algebras of Fell bundles, Rocky Mountain J. Math., 47 (2017), no. 2, 351-381.

3. F. Abadie, Sobre ações parciais, fibrados de Fell e grupóides, PhD Thesis, São Paulo, 1999.

4. F. Abadie, Enveloping Actions and Takai Duality for Partial Actions, J. Funct. Analysis, 197 (2003), (1), 14-67.

5. F. Abadie, On partial actions and groupoids, Proc. Am. Math. Soc., 132 (2003), (4), 1037-1047.

6. F. Abadie, Partial actions of discrete groups and related structures, Publ. Mat. Urug. 10 (2005), 1-9.

7. F. Abadie, A. Buss, D. Ferraro, Morita enveloping Fell bundles, Preprint, arXiv:1711.03013, (2017).

8. F. Abadie, M. Dokuchaev, R. Exel, J. J. Simón, Morita equivalence of partial group actions and globalization, Transaction AMS, 368 (2016), 4957-4992.

9. F. Abadie, D. Ferraro, Applications of ternary rings to $C^{*}$-algebras, Adv. Oper. Theory, 2, (2017), (3), 293-317.

10. F. Abadie, D. Ferraro, Equivalence of Fell bundles over groups, Preprint, arXiv:1711.02577, (2017).

11. F. Abadie, L. Martí Pérez, On the amenability of partial and enveloping actions, Proc. Amer. Math. Soc., 137 (2009), (11), 3689-3693.

12. J. N. Alonso Álvarez, J. M. Fernández Vilaboa, R. González Rodríguez, Crossed products over weak Hopf algebras related to cleft extensions and cohomology, Chin. Ann. Math., Ser. B 35, (2014), (2), 161-190.

13. J. N. Alonso Álvarez, J. M. Fernández Vilaboa, R. González Rodríguez, Cohomology of algebras over weak Hopf algebras, Homology Homotopy Appl., 16, (2014), (1), 341369.

14. J. N. Alonso Álvarez, J. M. Fernández Vilaboa, R. González Rodríguez, C. Soneira Calvo, Lax entwining structures, groupoid algebras and cleft extensions, Bull. Braz. Math. Soc. 45, No. 1, (2014), 133-178.

15. E. R. Alvares, M. M. S. Alves, E. Batista, Partial Hopf module categories, J. Pure Appl. Algebra, 217 (2013), 1517-1534.

16. E. R. Alvares, M. M. S. Alves, M. J. Redondo, Cohomology of partial smash products, J. Algebra, 482, (2017), 204-223.

17. M. M. S. Alves, E. Batista, Partial Hopf actions, partial invariants and a Morita context, J. Algebra Discrete Math. no. 3, (2009), 1-19.

18. M. M. S. Alves, E. Batista, Enveloping Actions for Partial Hopf Actions, Commun. Algebra, 38, (2010) 2872-2902. 
19. M. M. S. Alves, E. Batista, Globalization theorems for partial Hopf (co)actions and some of their applications, Contemp. Math. 537 (2011), 13-30.

20. M.M.S. Alves, E. Batista, M. Dokuchaev, A. Paques, Twisted partial actions of Hopf algebras, Israel J. Math. 197 (2013) 263-308 (arXiv:1111.1281).

21. M.M.S. Alves, E. Batista, M. Dokuchaev, A. Paques, Globalization of Twisted partial Hopf actions, J. Aust. Math. Soc., 101 (2016), 1-28 (arXiv:1503.00124).

22. M. M. S. Alves, E. Batista, J. Vercruysse, Partial representations of Hopf algebras, J. Algebra, 426, 15 (2015), 137-187. (arXiv: 1309.1659v2.)

23. P. Ara, R. Exel, Dynamical systems associated to separated graphs, graph algebras, and paradoxical decompositions, Advances Math., 252 (2014), 748-804.

24. P. Ara, R. Exel, $K$-theory for the tame $C^{*}$-algebra of a separated graph, J. Funct. Analysis, 269 (2015), 2995-3041.

25. P. Ara, R. Exel, T. Katsura, Dynamical systems of type $(m, n)$ and their $C^{*}$-algebras, Ergodic Theory Dynam. Systems, 33, (2013), No. 5, 1291-1325.

26. A. Artigue, Isolated sets, catenary Lyapunov functions and expansive systems, Topol. Methods Nonlinear Anal., 49, (1), (2017), 21-50.

27. J. Ávila, Partial Actions and Quotient Rings, Proyecciones J. Math., 30, (2011), No 2, 201-212.

28. J. Ávila, S. Buitrago, S. Zapata, The category of partial actions of groups: some constructions, Int. J. Pure Applied Math., 95, (2014), No. 1, 45-56.

29. J. Ávila, M. Ferrero, Closed and prime ideals in partial skew group rings of abelian groups, J. Algebra Appl. 10 (2011), no. 5, 961-978.

30. J. Ávila, M. Ferrero, J. Lazzarin, Partial actions and partial fixed rings, Commun. Algebra 38 (2010), no. 6, 2079-2091.

31. J. Ávila, L. Hernández-González, A. Ortiz-Jara, On partial orbits and stabilizers, Int. Electr. J. Pure Appl. Math., 8, (2014), No. 3, 101-106.

32. J. Ávila Guzmán, J. Lazzarin, A Morita Context Related to Finite Groups Acting Partially on a Ring, J. Algebra Discrete Math. no. 3, (2009), 49-60.

33. J. Ávila, J. Lazzarin, Anillos que son suma de ideales, Tumbaga, 1, (2011), 227-236.

34. J. Ávila, J. Lazzarin, Partial actions and power sets, Int. J. Math. Math. Sci., 2013, Article ID 376915, 4 p. (2013).

35. J. Ávila, J. Lazzarin, Partial skew group rings and applications to modules, JP J. Algebra Number Theory Appl., 35, (2014), No. 1, 15-23.

36. D. Azevedo, E. Campos, G. Fonseca, G. Martini, Partial (Co)Actions of Multiplier Hopf Algebras: Morita and Galois Theories, arXiv: 1709.08051, (2017).

37. D. Bagio, Partial actions of inductive groupoids on rings, Int. J. Math. Game Theory Algebra, 20, (2011), (3), 247-261.

38. D. Bagio, W. Cortes, M. Ferrero, A. Paques, Actions of inverse semigroups on algebras, Commun. Algebra 35 (12) (2007), 3865-3874.

39. D. Bagio, J. Lazzarin, A. Paques, Crossed products by twisted partial actions: separability, semisimplicity and Frobenius properties, Commun. Algebra 38 (2010), 496-508.

40. D. Bagio, D. Flores, A. Paques, Partial actions of ordered groupoids on rings, J. Algebra Appl. 9 (2010), (3), 501-517.

41. D. Bagio, A. Paques, Partial groupoid actions: globalization, Morita theory and Galois theory, Comm. Algebra, 40, (2012), 3658-3678.

42. D. Bagio, H. Pinedo, Globalization of partial actions of groupoids on nonunital rings, J. Algebra Appl. 15, No. 5 (2016), 1650096 (16 pages).

43. D. Bagio, H. Pinedo, On the separability of the partial skew groupoid ring, São Paulo J. Math. Sci., 11, (2017), 370-384.

44. A. Baraviera, W. Cortes, M. Soares, Simplicity of Partial Crossed Products, Preprint.

45. E. Batista, Partial actions: What they are and why we care, Bull. Belg. Math. Soc. Simon Stevin, 24, (2017), (1), 35-71 (arXiv:1604.06393v1). 
46. E. Batista, S. Caenepeel, J. Vercruysse, Hopf Categories, Algebr. Represent. Theory, 19, (2016), (5), 1173-1216 (arXiv:1503.05447).

47. E. Batista, A. D. M. Mortari, M. M. Teixeira, Cohomology for partial actions of Hopf algebras, Preprint, arXiv:1709.03910, (2017).

48. E. Batista, J. Vercruysse, Dual Constructions for Partial Actions of Hopf Algebras, J. Pure Appl. Algebra 220 (2016), (2), 518-559 (arXiv:1403.1399).

49. L. Bemm, W. Cortes, Ring Theoretic Properties of Partial Crossed products and related themes, arXiv:1603.08404, (2016).

50. L. Bemm, W. Cortes, S. Della Flora, M. Ferrero, Partial crossed product and Goldie rings, Comm. Algebra, 43 (9) (2015), 3705 - 3724.

51. L. Bemm, M. Ferrero, Globalization of partial actions on semiprime rings, J. Algebra Appl., 12 (2013), (4), Paper No. 1250202, 9 p.

52. V. Beuter, D. Gonçalves, Partial crossed products as equivalence relation algebras, Rocky Mountain J. Math., 46, (2016), No. 1, 85-104.

53. V. Beuter, D. Gonçalves, The interplay between Steinberg algebras and partial skew rings, arXiv:1706.00127, (2017).

54. V. Beuter, D. Gonçalves, J. Öinert, D. Royer, Simplicity of skew inverse semigroup rings with an application to Steinberg algebras, Preprint, arXiv:1708.04973, (2017).

55. J. Bichon, C. Kassel, The lazy homology of a Hopf algebra, J. Algebra, 323, (9), (2010), 2556-2590.

56. J.-C. Birget, The Groups of Richard Thompson and Complexity, Internat. J. Algebra Comput., 14 (2004), Nos. 5\& 6, 569-626.

57. J.-C. Birget, J. Rhodes, Almost finite expansions of arbitrary semigroups, J. Pure Appl. Algebra, 32 (1984), 239-287.

58. J.-C. Birget, J. Rhodes, Group theory via global semigroup theory, J. Algebra, 120 (1989), 284-300.

59. R. J. Blattner, S. Montgomery, A duality theorem for Hopf module algebras, J. Algebra 95 (1985), 153-172.

60. G. Boava, Caracterizações da $C^{*}$-álgebra gerada por uma Compreessão Aplicadas a Cristais e Quasecristais, Master Thesis, Florianópolis, 2007.

61. G. Boava, R. Exel, Partial crossed product description of the $\mathrm{C}^{*}$-algebras associated with integral domains, Proc. AMS, 141, No. 7, (2013), 2439-2451.

62. G. Böhm, The weak theory of monads, Adv. Math., 225, (1), (2010), 1-32.

63. G. Böhm, T. Brzezinski, Cleft extensions of Hopf algebroids, Appl. Categorical Structures, 14, no.5-6, (2006), 431-469.

64. G. Böhm, D. Stefan, (Co) cyclic (co) homology of bialgebroids: an approach via (co) monads, Comm. Math. Physics 282, (1), (2008), 239-286.

65. G. Böhm, J. Vercruysse, Morita theory for coring extensions and cleft bicomodules, Adv. Math., 209, (2007), 611-648.

66. T. Brzeziński, Descent cohomology and corings, Commun. Algebra, 36 (2008), (5), 1894-1900.

67. A. Buss, R. Exel, Twisted actions and regular Fell bundles over inverse semigroups, Proc. Lond. Math. Soc., (3) 103, No. 2, (2011), 235-270.

68. A. Buss, R. Exel, Inverse semigroup expansions and their actions on $C^{*}$-algebras, Illinois J. Math., 56 (2012), 1185-1212.

69. S. Caenepeel, E. De Groot, Galois corings applied to partial Galois theory, Proc. ICMA-2004, Kuwait Univ. (2005), 117-134.

70. S. Caenepeel, E. De Groot, Galois theory for weak Hopf algebras, Rev. Roumaine Math. Pures Appl., 52 (2007), 151-176.

71. S. Caenepeel, C. Janssen, Partial entwining structures, arXiv:0610524 (2007).

72. S. Caenepeel, K. Janssen, Partial (Co)Actions of Hopf Algebras and Partial HopfGalois Theory, Commun. Algebra, 36, (2008), 2923-2946. 
73. T. M. Carlsen, Cuntz-Pimsner $C^{*}$-algebras associated with subshifts, Internat. J. Math., 19, (2008), 47-70.

74. T. M. Carlsen, N. S. Larsen, Partial actions and KMS states on relative graph $C^{*}$ algebras, J. Funct. Anal., 271, (8), (2016), 2090-2132.

75. T. M. Carlsen, K. Matsumoto, Some remarks on the $C^{*}$-algebras associated with subshifts, Math. Scand., 95 (2004), 145-160.

76. H. Cartan, S. Eilenberg, Homological algebra, Princeton Mathematical Series, 19. Princeton, New Jersey: Princeton University Press, (1956).

77. P. A. A. B. Carvalho, W. Cortes, M. Ferrero, Partial skew group rings over polycyclic by finite groups, Algebr. Represent. Theory, 14, (3), (2011), 449-462.

78. F. Castro, A. Paques, G. Quadros, A. Sant'Ana, Partial actions of weak Hopf algebras: Smash product, globalization and Morita theory, J. Pure Appl. Algebra, 219, (2015), $5511-5538$

79. F. Castro, A. Paques, G. Quadros, A. Sant'Ana, Partial bi(co)module algebras, globalizations, and partial (L,R)-smash products, Preprint, arXiv:1505.05019v1, (2015).

80. F. Castro and G. Quadros, Globalizations for partial (co)actions on coalgebras, Preprint, arXiv:1510.01388v1, (2015).

81. F. Castro and G. Quadros, Galois Theory for Partial Comodule Coalgebras, Preprint.

82. R. Cavalheiro, A. Sant'Ana, On the semiprimitivity and the semiprimality problems for partial smash products, Preprint, arXiv:1510.05013, (2015).

83. R. Cavalheiro, A. Sant'Ana, On partial $H$-radicals of Jacobson type, São Paulo J. Math. Sci., 10, (2016), (1), 140-163.

84. S. U. Chase, A. Rosenberg, Amitsur cohomology and the Brauer group, Mem. Am. Math. Soc., 52, (1965), 34-79.

85. S.U. Chase, D.K. Harrison, A. Rosenberg, Galois theory and Galois cohomology of commutative rings, Mem. Amer. Math. Soc., 52, (1965), 15-33.

86. Q.-G. Chen, D.-G. Wang, The category of partial Doi-Hopf modules and functors, Rend. Semin. Mat. Univ. Padova, 129, (2013), 189-204.

87. Q.-G. Chen, D.-G. Wang, Partial group (co)actions of Hopf group coalgebras, Filomat, 28, (2014), (1), 131-151.

88. Q.-G. Chen, D.-G. Wang, X. Kang, Twisted partial coactions of Hopf algebras Frontiers of Mathematics in China, 12, (2017), (1), 63-86.

89. K. Choi, Green's $\mathcal{D}$-relation of Birget-Rhodes expansions and partial group algebras, Kyungpook Math. J. , 44, (2004), No. 3, 443-448.

90. K. Choi, Y. Lim, Inverse monoids of Möbius type, J. Algebra 223, (1), (2000), 283-294.

91. K. Choi, Y. Lim, Birget Rhodes expansion of groups and inverse monoids of Möbius type, Internat. J. Algebra Comput. 12, (4), (2002), 525 - 533.

92. K. Choi, Y. Lim, Greens equivalences of Birget-Rhodes expansions of finite groups, Bull. Korean Math. Soc., 43, (2), (2006), 353-375.

93. K. Choi, Y. Lim, Transitive partial actions of groups, Period. Math. Hung. , 56, (2008), No. 2, 169-181.

94. C. Cibils and A. Solotar, Galois coverings, Morita equivalence and smash extensions of categories over a field, Doc. Math., 11, (2006), 143-159.

95. L. O. Clark, C. Farthing, A. Sims, M. Tomforde, A groupoid generalization of Leavitt path algebras, Semigroup Forum, 89 (2014), 501-517.

96. M. Cohen, S. Montgomery, Group-graded rings, smash products, and group actions, Trans. AMS, 282, (1984), 237-258.

97. C. Cornock, V. Gould, Proper restriction semigroups and partial actions, J. Pure Appl. Algebra, 216, (2012), 935-949.

98. W. Cortes, Partial Skew Polynomial Rings and Jacobson Rings, Commun. Algebra, 38, (2010), (4), 1526-1548.

99. W. Cortes, On partial skew Armendariz rings, Algebra Discrete Math., 11, (2011), (1), 23-45. 
100. W. Cortes, Prime Goldie Ideals in Partial Skew Polynomial Rings, Houston J. Math., 40, (2014), 1021-1033.

101. W. Cortes, M. Ferrero, Partial skew polynomial rings: Prime and Maximal ideals, Commun. Algebra, 35, (2007), (4), 1183-1200.

102. W. Cortes, M. Ferrero, Globalizations of Partial Actions on Semiprime Rings, Contemp. Math., 499, (2009), 27-35.

103. W. Cortes, M. Ferrero, L. Gobbi, Quasi-duo Partial skew polynomial rings, Algebra Discrete Math., 12, (2011), (2), 53-63.

104. W. Cortes, M. Ferrero, Y. Hirano, H. Marubayashi, Partial Skew polynomial rings over semisimple Artinian rings, Commun. Algebra, 38, (5), (2010), 1663-1676.

105. W. Cortes, M. Ferrero, E. Marcos, Partial Actions on Categories, Commun. Algebra, 44, (2016), (7), 2719-2731.

106. W. Cortes, M. Ferrero, H. Marubayashi, Partial Skew polynomial Rings and Goldie Rings, Commun. Algebra, 36, (2008), (11), 4284-4295.

107. W. Cortes, L. Gobbi, Bezout duo and distributive partial skew power series rings, Publ. Math. (Debrecen) 83, (2013), (4), 547-568.

108. W. Cortes, E. Marcos, Description of Partial actions, Preprint, arXiv:1708.01330, (2017)

109. W. Cortes, S. Ruiz, Goldie twisted partial skew power series rings, Preprint, arXiv:1709.09791, (2017).

110. T. Coulbois, Free products, profinite topology and finitely generated subgroups, Internat. J. Algebra Comput., 11, (2001), 171-184.

111. T. Coulbois, Partial actions of groups on relational structures: a connection between model theory and profinite topology, In: Semigroups, Algorithms, Automata and Languages, World Scientific, Singapore, 2002, 349-361.

112. J. Crisp, M. Laca, Boundary quotients and ideals of Toeplitz $C^{*}$-algebras of Artin groups, J. Funct. Anal. 242, (1), (2007), 127-156.

113. J. Cuntz, W. Krieger, A class of $C^{*}$-algebras and topological Markov chains, Inventiones Math., 56 (1980), 251-268.

114. P. Demeneghi, The Ideal Structure of Steinberg Algebras, Preprint, arXiv:1710.09723, (2017).

115. M. Dokuchaev, Partial actions, crossed products and partial representations, Resenhas IME-USP, 5, (4), (2002), 305-327.

116. M. Dokuchaev, Partial actions: a survey, Contemp. Math., 537, (2011), 173-184.

117. M. Dokuchaev, R. Exel, Associativity of crossed products by partial actions, enveloping actions and partial representations, Trans. Am. Math. Soc., 357, (2005), 1931-1952.

118. M. Dokuchaev, R. Exel, Partial actions and subshifts, J. Funct. Analysis, 272, (2017), 5038-5106.

119. M. Dokuchaev, R. Exel, The ideal structure of algebraic partial crossed products, Proc. London Math. Soc., 115, (1), (2017), 91-134.

120. M. Dokuchaev, R. Exel, P. Piccione, Partial representations and partial group algebras, J. Algebra, 226, (1), (2000), 251-268.

121. M. Dokuchaev, R. Exel, J. J. Simón, Crossed products by twisted partial actions and graded algebras, J. Algebra, 320, (8), (2008), 3278-3310.

122. M. Dokuchaev, R. Exel, J. J. Simón, Globalization of twisted partial actions, Trans. Am. Math. Soc., 362, (8), (2010), 4137-4160.

123. M. Dokuchaev, M. Ferrero, A. Paques, Partial Actions and Galois Theory, J. Pure Appl. Algebra, 208, (1), (2007), 77-87.

124. M. Dokuchaev, M. Khrypchenko, Partial cohomology of groups, J. Algebra, 427, (2015), 142-182. 
125. M. Dokuchaev, M. Khrypchenko, Twisted partial actions and extensions of semilattices of groups by groups, Int. J. Algebra Comput., 27, (7), (2017), 887-933, arXiv: $1602.02424 \mathrm{v} 1$.

126. M. Dokuchaev, M. Khrypchenko, Partial cohomology of groups and extensions of semilattices of abelian groups, J. Pure Appl. Algebra (to appear), arXiv:1705.09654 (2017).

127. M. Dokuchaev, M. Khrypchenko, J. J. Simón, Globalization of partial cohomology of groups, arXiv:1706.02546, (2017).

128. M. Dokuchaev, H. G. G. de Lima, H. Pinedo, Partial representations and their domains, Rocky Mountain J. Math., 47, (8), (2017), 2565-2604.

129. M. Dokuchaev, B. Novikov, Partial projective representations and partial actions, $J$. Pure Appl. Algebra, 214, (2010), 251-268.

130. M. Dokuchaev, B. Novikov, Partial projective representations and partial actions II, J. Pure Appl. Algebra, 216, (2012), 438-455.

131. M. Dokuchaev, B. Novikov, H. Pinedo, The partial Schur Multiplier of a group, J. Algebra, 392, (2013), 199-225.

132. M. Dokuchaev, B. Novikov, G. Zholtkevych, Partial actions and automata, Algebra Discrete Math., 11, (2), (2011), 51-63.

133. M. Dokuchaev, A. Paques, H. Pinedo, Partial Galois cohomology and related homomorphisms, Preprint.

134. M. Dokuchaev, A. Paques, H. Pinedo, J. I. Rocha, Partial generalized crossed products and a seven-term exact sequence, Preprint.

135. M. Dokuchaev, C. Polcino Milies, Isomorphisms of partial group rings, Glasg. Math. J., 46, (1), (2004), 161-168.

136. M. Dokuchaev, A. del Río, J. J. Simón, Globalizations of partial actions on non unital rings, Proc. Am. Math. Soc., 135, (2), (2007), 343-352.

137. M. Dokuchaev, N. Sambonet, Schur's theory for partial projective representations, Preprint, arXiv:1711.06739, (2017).

138. M. Dokuchaev, J. J. Simón, Invariants of partial group algebras of finite $p$-groups, Contemp. Math. 499 (2009), 89-105.

139. M. Dokuchaev, J. J. Simón, Isomorphisms of partial group rings, Commun. Algebra, 44, (2016), 680-696.

140. M. Dokuchaev, N. Zhukavets, On finite degree partial representations of groups, J. Algebra, 274, (1), (2004), 309-334.

141. A. P. Donsig, A. Hopenwasser, Analytic partial crossed products, Houston J. Math., 31, (2), (2005), 495-527.

142. A. Dooms, P. M. Veloso, Normalizers and free groups in the unit group of an integral semigroup ring, J. Algebra Appl., 6, (4), (2007), 655-669.

143. C. Z. Du, J. F. Lin, Morita context, partial Hopf Galois extensions and partial entwining structure, Math. Notes 95, (1), (2014), 43-52.

144. E. G. Effros, Transformations groups and $C^{*}$-algebras, Ann. Math., 81, (1965), 3855.

145. E. G. Effros, F. Hahn, Locally compact transformation groups and $C^{*}$-algebras, Memoirs of the American Math. Soc., no. 75, (1967).

146. R. Exel, Circle actions on $C^{*}$-algebras, partial automorphisms and generalized Pimsner-Voiculescu exact sequences, J. Funct. Anal., 122, (3), (1994), 361-401.

147. R. Exel, The Bunce-Deddens algebras as crossed products by partial automorphisms, Bol. Soc. Brasil. Mat. (N.S.), 25, (1994), 173-179.

148. R. Exel, Approximately finite $\mathrm{C}^{*}$-algebras and partial automorphisms, Math. Scand., 77, (1995), 281-288.

149. R. Exel, Twisted partial actions: a classification of regular $C^{*}$-algebraic bundles, Proc. London Math. Soc., 74, (3), (1997), 417-443.

150. R. Exel, Amenability for Fell Bundles, J. Reine Angew. Math., 492, (1997), 41-73. 
151. R. Exel, Partial actions of groups and actions of inverse semigroups, Proc. Am. Math. Soc., 126, (12), (1998), 3481-3494.

152. R. Exel, Partial representations and amenable Fell bundles over free groups, Pacific J. Math., 192, (1), (2000), 39-63.

153. R. Exel, Hecke algebras for protonormal subgroups, J. Algebra, 320, (2008), 17711813.

154. R. Exel, Inverse semigroups and combinatorial $C^{*}$-algebras, Bull. Braz. Math. Soc., New Series, 39, (2), (2008), 191-313.

155. R. Exel, Partial Dynamical Systems, Fell Bundles and Applications, Mathematical Surveys and Monographs, vol. 224, American Mathematical Society, 2017.

156. R. Exel, T. Giordano and D. Gonçalves, Enveloping algebras of partial actions as groupoid $C^{*}$-algebras, J. Operator Theory, 65, (2011), 197-210.

157. R. Exel, M. Laca, Cuntz-Krieger Algebras for Infinite Matrices, J. Reine Angew. Math. ,512, (1999), 119-172.

158. R. Exel, M. Laca, The K-Theory of Cuntz-Krieger Algebras for Infinite Matrices, K-Theory, 19, (2000), 251-268.

159. R. Exel, M. Laca, Partial dynamical systems and the KMS condition, Comm. Math. Phys., 232, (2), (2003), 223-277.

160. R. Exel, M. Laca, J. Quigg, Partial dynamical systems and $C^{*}$-algebras generated by partial isometries, J. Operator Theory, 47, (1), (2002), 169-186.

161. R. Exel, E. Pardo, Representing Kirchberg algebras as inverse semigroup crossed products, Preprint arXiv:1303.6268, (2013).

162. R. Exel, C. Starling, Self-Similar Graph $C^{*}$-Algebras and Partial Crossed Products, J. Operator Theory, 75, (2016), 299-317.

163. R. Exel, F. Vieira, Actions of inverse semigroups arising from partial actions of groups, J. Math. Anal. Appl., 363, (2010), 86-96.

164. J. M. Fernández Vilaboa, R. González Rodríguez, A. B. Rodríguez Raposo, Preunits and weak crossed products, J. Pure Appl. Algebra, 213, (2009), 2244-2261.

165. J. M. Fernández Vilaboa, R. González Rodríguez, A. B. Rodríguez Raposo, Partial and unified crossed products are weak crossed products, Contemp. Math. AMS (Hopf Algebras and Tensor Categories) 585, (2013), 261-274 arXiv:1110.6724.

166. J. M. Fernández Vilaboa, R. González Rodríguez, A. B. Rodríguez Raposo, Iterated weak crossed products, arXiv:1503.01585, (2015).

167. J. M. Fernández Vilaboa, R. González Rodríguez, A. B. Rodríguez Raposo, Equivalences for weak crossed products, Commun. Algebra, 44, (10), (2016), 4519-4545.

168. D. Ferraro, Construction of Globalization for Partial Actions on Rings, Algebras, $C^{*}$-Algebras and Hilbert Bimodules, to appear in Rocky Mountain J. Math, (arXiv:1209.4094).

169. M. Ferrero, Partial actions of groups on semiprime rings, Groups, rings and group rings, Lect. Notes Pure Appl. Math., 248, Chapman \& Hall/CRC, Boca Raton, FL, (2006), 155-162.

170. M. Ferrero, Partial actions of groups on algebras, a survey, São Paulo J. Math. Sci. 3, (1), (2009), 95-107.

171. M. Ferrero, J. Lazzarin, Partial actions and partial skew group rings, J. Algebra, 319, (12), (2008), 5247-5264.

172. J. R. Fisher, A Jacobson radical for Hopf module algebras, J. Algebra, 34, (1975), $217-231$.

173. D. Flôres, A. Paques, On the structure of skew groupoid rings which are Azumaya, Algebra Discrete Math., 16, (1), (2013), 71-80.

174. D. Flôres, A. Paques, Duality for groupoid (co)actions, Commun. Algebra, 42, (2014), 637-663.

175. J. Fountain, G. M. S. Gomes, The Szendrei expansion of a semigroup, Mathematika, 37, (2), (1990), 251-260. 
176. D. Freitas, A. Paques, On partial Galois Azumaya extensions, Algebra Discrete Math., 11, (2011), 64-77.

177. J. L. García, J. J. Simón, Morita equivalence for idempotent rings, J. Pure Appl. Algebra, 76, (1), (1991), 39-56.

178. N. D. Gilbert, Actions and expansions of ordered groupoids, J. Pure Appl. Algebra, 198, (2005), 175-195.

179. T. Giordano, D. Gonçalves, C. Starling, Bratteli-Vershik models for partial actions of $\mathbb{Z}$, Preprint, arXiv:1611.04389, (2016).

180. T. Giordano, A. Sierakowski, Purely infinite partial crossed products, J. Funct. Anal. 266, (9), (2014), 5733-5764.

181. J. Gómez, H. Pinedo, C. Uzcátegui, The open mapping principle for partial actions of polish groups, J. Math. Anal. Appl, 462, (2018), 337-346 (arXiv:1709.01977).

182. J. Gómez, H. Pinedo, C. Uzcátegui, On the continuity of partial actions of Hausdorff groups on metric spaces, arXiv:1708.09474, (2017).

183. D. Gonçalves, Simplicity of partial skew group rings of abelian groups, Canad. Math. Bull., 57, (3), (2014), 511-519.

184. D. Gonçalves, J. Oinert, D. Royer, Simplicity of partial skew group rings with applications to Leavitt path algebras and topological dynamics, J. Algebra, 420, (2014), 201-216.

185. D. Gonçalves, D. Royer, $C^{*}$-algebras associated to stationary ordered Bratteli diagrams, Houston J. Math., 40 (1), (2014), 127-143.

186. D. Gonçalves, D. Royer, Leavitt path algebras as partial skew group rings, Commun. Algebra, 42, (2014), 127-143.

187. D. Gonçalves, D. Royer, Ultragraphs and shifts spaces over infinite alphabets, Preprint, arXiv:1510.04649, (2015).

188. D. Gonçalves, D. Royer, Infinite alphabet edge shift spaces via ultragraphs and their $C^{*}$-algebras, Preprint, arXiv:1703.05069, (2017).

189. D. Gonçalves, D. Royer, Simplicity and chain conditions for ultragraph Leavitt path algebras via partial skew group ring theory, Preprint, arXiv:1706.03628, (2017).

190. Gonçalves, D., Yoneda, G., Free path groupoid grading on Leavitt path algebras, Int. J. Algebra Comput., 26, (6), (2016), 1217-1235.

191. G. Gonçalves de Castro, D. Gonçalves, KMS and ground states on ultragraph $C^{*}$ algebras, Preprint, arXiv:1710.09337, (2017).

192. J. Gonçalves Lopes, On strongly associative group algebras, Comm. Algebra, 36, (2008), 478-492.

193. J. Gonçalves Lopes, On strongly associative group algebras of $p$-solvable groups, $J$. Algebra Appl. 14.6 (2015).

194. J. Gonçalves Lopes, Characterizations of Strongly Associative Group Algebras, Intern. J. Algebra, 9 (2015), no. 4, 165-178.

195. J. Gonçalves Lopes, On strongly associative semigroup algebras, Preprint, (2016).

196. V. Gould, Notes on restriction semigroups and related structures, preprint at, http://www-users.york.ac.uk/ varg1/restriction.pdf.

197. V. Gould, C. Hollings, Partial actions of inverse and weakly left $E$-ample semigroups, J. Aust. Math. Soc., 86, (3), (2009), 355-377.

198. V. Gould, C. Hollings, Actions and partial actions of inductive constellations, Semigroup Forum, 82, (1), (2011), 35-60.

199. C. Hollings, Partial actions of monoids, Semigroup Forum, 75, (2), (2007), 293-316.

200. C. Hollings, From right PP monoids to restriction semigroups: a survey, Eur. J. Pure Appl. Math., 2, (1), (2009), 21-57.

201. X.-L. Jiang, G. Szeto, Galois extensions induced by a central idempotent in a partial Galois extension, Intern. J. Algebra, 8, (11), (2014), 505-510.

202. X.-L. Jiang, G. Szeto, The group idempotents in a partial Galois extension, Gulf J. Math., 3, (2015), (3), 42-47." 
203. T. Kanzaki, On generalized crossed product and Brauer group, Osaka J. Math (1968) 5: $175-188$.

204. J. Kellendonk, M. V. Lawson, Tiling semigroups, J. Algebra, 224, (2000), 140-150.

205. J. Kellendonk, M. V. Lawson, Partial actions of groups, Internat. J. Algebra Comput., 14, (1), (2004), 87-114, (preprint 2000).

206. M. Kennedy, C. Schafhauser, Noncommutative boundaries and the ideal structure of reduced crossed products, Preprint, arXiv:1710.02200, (2017).

207. D. Kerr, $C^{*}$-algebras and topological dynamics: finite approximation and paradoxicality, CRM Advanced course books, Birkhauser, to appear; http://www.math.tamu.edu/ kerr/.

208. D. Kerr, P. W. Nowak, Residually finite actions and crossed products, Ergodic Theory Dynam. Systems, 32, (2012), 1585-1614.

209. M. Khrypchenko, Partial actions and an embedding theorem for inverse semigroups, Preprint, arXiv:1702.00059, (2018).

210. M. Khrypchenko, B. Novikov, Reflectors and globalizations of partial actions of groups, J. Aust. Math. Soc. (to appear), arXiv:1602.05504.

211. M. Khoshkam, G. Skandalis, Regular representation of groupoid $C^{*}$-algebras and applications to inverse semigroups, J. Reine Angew. Math., 546, (2002), 47-72.

212. F. Kraken, P. Quast, T. Timmermann, Partial actions of $C^{*}$-quantum groups, $B a-$ nach J. Math. Anal., (2018), doi:10.1215/17358787-2017-0056, arXiv:1703.06546.

213. G. Kudryavtseva, Partial monoid actions and a class of restriction semigroups, J. Algebra, 429, (2015), 342-370.

214. J.-M. Kuo, G. Szeto, The structure of a partial Galois extension, Monatsh. Math, 175, (4), (2014), 565-576.

215. J.-M. Kuo, G. Szeto, The structure of a partial Galois extension. II, J. Algebra Appl. 15, No. 4, Article ID 1650061, 12 p. (2016).

216. J.-M. Kuo, G. Szeto, Partial group actions and partial Galois extensions, Monatsh. Math, 185, (2018), 287-306.

217. B. K. Kwaśniewski, R. Meyer, Aperiodicity, topological freeness and pure outerness: from group actions to Fell bundles, Studia Math., 241, (2018), 257-302.

218. H. Lausch, Cohomology of inverse semigroups, J. Algebra, 35 , (1975), 273-303.

219. M. V. Lawson, Inverse semigroups. The theory of partial symmetries, World Scientific Publishing Co., Inc., River Edge, NJ, 1998.

220. M. V. Lawson, S. Margolis, B. Steinberg, Expansions of inverse semigroups, J. Aust. Math. Soc., 80, (2), (2006), 205-228.

221. W. G. Leavitt, The module type of a ring, Trans. Amer. Math. Soc., 103, (1962), $113-130$.

222. A. V. Lebedev, Topologically free partial actions and faithful representations of crossed products, Funct. Anal. Appl. 39, No. 3, 207-214 (2005); translation from Funkts. Anal. Prilozh. 39, No. 3, 54-63 (2005).

223. J. Leech, $\mathcal{H}$-coextensions of monoids, Mem. Amer. Math. Soc., 157, (1975), 1-66.

224. X. Li, Partial transformation groupoids attached to graphs and semigroups, Prerpint, arXiv:1603.09165, (2016).

225. X. Li, M. D. Norling, Independent resolutions for totally disconnected dynamical systems II: $C^{*}$-algebraic case, J. Operator Theory, 75, (1), (2016), 163-193.

226. H. G. de Lima, H. Pinedo, On the total component of the partial Schur Multiplier, J. Aust. Math. Soc. 100, No. 3, (2016), 374-402.

227. C. Limin, Structure of $E^{*}$-unitary categorical inverse semigroups, J. Math. Res. Expo. 26, No. 2, (2006), 239-246.

228. D. Lind and B. Marcus, An introduction to symbolic dynamics and coding, Cambridge Univ. Press, 1999.

229. M. Loganathan, Cohomology of inverse semigroups, J. Algebra, 70, ( 1981), 375-393. 
230. Ch. Lomp, Duality for partial group actions, Internat. Electronic J. Algebra, 4, (2008), 53-62.

231. K. Matsumoto, On automorphisms of $\mathrm{C}^{*}$-algebras associated with subshifts, J. Operator Theory, 44, (2000), 91-112

232. K. McClanahan, K-theory for partial crossed products by discrete groups, J. Funct. Anal., 130, (1995), (1), 77-117.

233. M. G. Megrelishvili, L. Schröder, Globalization of confluent partial actions on topological and metric spaces, Topology Appl., 145, (2004), 119-145.

234. I. Mikhailova, B. Novikov, G. Zholtkevych, Protoautomata as models of systems with data accumulation, Proceedings of the 9th International Conference ICTERI, pp. 582-589, CEUR-WS (2013).

235. D. Milan, B. Steinberg, On inverse semigroup $C^{*}$-algebras and crossed products, Groups Geom. Dyn., 8, (2), (2014), 485-512.

236. Y. Nakagami, M. Takesaki, Duality for crossed products of Von Neumann algebras, Lecture Notes in Math., 731, Springer Verlag (1979).

237. M. D. Norling, The $K$-theory of some reduced inverse semigroup $C^{*}$-algebras, Math. Scand., 117, (2), (2015), 186-202.

238. B. V. Novikov, On multipliers of semigroups, 26 pp. VINITI, Moscow, 1976 (in Russian).

239. B. V. Novikov, On projective representations of semigroups, Dopovidi AN USSR, 1979, N6, 472-475 (in Russian). [MR 80i:20041].

240. B. V. Novikov, On 0-cohomology of semigroups, In: "Theor. and Appl. Quest. of Diff. Equat. and Algebra". Kiev, Naukova dumka, (1978), 185-188 (in Russian).

241. B. V. Novikov, Semigroup cohomology and applications, In: Algebra - Representation Theory (ed. K. W. Roggenkamp and M. Ştefănescu), Kluwer, (2001), 219-234.

242. B. Novikov, I. Perepelytsya, G. Zholtkevych, Pre-automata as Mathematical Models of Event Flows Recognisers, Proceedings of the 7th International Conference ICTERI, pp. 41-50, CEUR-WS (2011).

243. B. Novikov, H. Pinedo, On components of the partial Schur multiplier, Comm. Algebra, 42, (2014), (6), 2484-2495.

244. P. Nystedt, Partial Category Actions on Sets and Topological Spaces, Commun. Algebra, 46, (2), (2018), 671-683, arXiv:1602.07541.

245. P. Nystedt, J. Öinert, Simple Semigroup Graded Rings, J. Algebra Appl. 14, (7), article id. 1550102, (2015) 10 p., arXiv:1308.3459.

246. P. Nystedt, J. Öinert, Outer Partial Actions and Partial Skew Group Rings, Canad. J. Math., 67, (2015), 1144-1160.

247. P. Nystedt, J. Öinert, Pinedo, H., Atinian and noetherian partial skew groupoid rings, J. Algebra, 503, (2018), 433-452 (arXiv:1603.02237v1).

248. P. Nystedt, J. Öinert, Pinedo, H., Epsilon-strongly graded rings, separability and simplicity, Preprint, arXiv:1606.07592, (2016).

249. L. O'Carroll, Strongly E-reflexive inverse semigroups, Proc. Edinburgh Math. Soc., 20, (2), (1977), 339-354.

250. A. Paques, Morita, Galois, and the trace map: a survey, São Paulo J. Math. Sciences, 10, (2), (2016), 372-383.

251. A. Paques, Galois theories: a survey, Preprint.

252. A. Paques, V. Rodrigues, A. Sant'Ana, Galois correspondences for partial Galois Azumaya extensions, J. Algebra Appl., 10, (5), (2011), 835-847.

253. A. Paques, A. Sant'Ana, When is a crossed product by a twisted partial action is Azumaya?, Comm. Algebra, 38, (2010), 1093-1103.

254. A. Paques, T. Tamusiunas, A Galois-Grothendieck-type correspondence for groupoid actions, Algebra Discrete Math., 17, (1), (2014), 80-97. 
255. I. D. Perepelytsya, G. M. Zholtkevych, Hierarchic decomposition of pre-machines as models of software system components, Syst. upravl. navig. i zv'iazku, 20, (4), (2011), 233-238.

256. M. Petrich, N. R. Reilly, A representation of E-unitary inverse semigroups, Q. J. Math., Oxf. II., Ser. 30, (1979), 339-350.

257. H. Pinedo, On elementary domains of partial projective representations of groups, Algebra Discrete Math., 15, (2013), (1), 63-82.

258. H. Pinedo, The partial Schur multiplier of $S_{3}$, Int. J. Math. Game Theory Algebra, 22, (2014), (4), 405-417.

259. H. Pinedo, Partial projective representations and the partial Schur multiplier: a survey, Bolet ín de Matemáticas, 22, (2015), (2), 167-175.

260. H. Pinedo, The total component of the partial Schur multiplier of the elementary abelian 3-group, Rev. Colomb. Mat., 50, (1), (2016), 75-83.

261. H. Pinedo, On the total component and the torsion part of the partial Schur multiplier, Commun. Algebra 45, (3), (2017), 954-966.

262. H. Pinedo, C. Uzcátegui, Polish globalization of polish group partial actions, Math. Log. Quart. (to appear), 63, (6), (2017), 481-490, arXiv:1602.06433v1.

263. H. Pinedo, C. Uzcátegui, Borel globalization of partial actions of polish groups, Arch. Math. Logic (to appear), arXiv:1702.02611.

264. J. C. Quigg, I. Raeburn, Characterizations of crossed Products by Partial Actions, J. Operator Theory, 37, (1997), 311-340.

265. J. N. Renault, D. P. Williams, Amenability of groupoids arising from partial semigroup actions and topological higher rank graphs, Trans. Am. Math. Soc., 369, (4), (2017), 2255-2283.

266. J. Renshaw, Inverse semigroups acting on graphs, in Semigroups and Languages, World Scientific, River Edge, NJ, 2004, pp. 212-239.

267. M. Rordam, A. Sierakowski, Purely infinite $C^{*}$-algebras arising from crossed products, Ergodic Theory Dynam. Systems, 32, (2012), 273-293.

268. J. J. Rotman, An Introduction to Homological Algebra, 2nd edn., Universitext, Springer, New York, 2009.

269. J. L. Sauvageot, Ideaux primitifs de certains produits croises, Math. Ann., 231, (1977), 61-76.

270. A. V. Sidorov, Radicals of $H$-module algebras, Algebra $i$ Logika, 28, (6), (1989), 705-721.

271. N. Sieben, $C^{*}$-crossed products by partial actions and actions of inverse semigroups, J. Aust. Math. Soc., Ser. A, 63, (1), (1997), 32-46.

272. N. Sieben, $C^{*}$-crossed products by twisted inverse semigroup actions, 39, J. Operator Theory, (1998), (2), 361-393.

273. N. Sieben, Morita equivalence of $C^{*}$-crossed products by inverse semigroup actions and partial actions, Rocky Mountain J. Math., 31, (2001), (2), 661-686.

274. C. Soneira Calvo, Invertible lax entwining structures and $\mathcal{C}$-cleft extensions, In: Gueye C., Molina M. (eds) Non-Associative and Non-Commutative Algebra and Operator Theory, Springer Proceedings in Mathematics \& Statistics, 160, (2016), 239-254.

275. B. Steinberg, Inverse semigroup homomorphisms via partial group actions, Bull. Aust. Math. Soc., 64, (1), (2001), 157-168.

276. B. Steinberg, Partial actions of groups on cell complexes, Monatsh. Math., 138, (2), (2003), 159-170.

277. B. Steinberg, A groupoid approach to discrete inverse semigroup algebras, Adv. Math., 223, (2), (2010), 689-727.

278. M. E. Sweedler, Cohomology of algebras over Hopf algebras, Trans. Am. Math. Soc., 133, (1968), 205-239.

279. M. B. Szendrei, A note on Birget-Rhodes expansion of groups, J. Pure Appl. Algebra, 58, (1989), 93-99. 
280. A. Van Daele, Multiplier Hopf algebras, Trans. Am. Math. Soc., 342, (1994), (2), 917-932.

281. F. Vieira, $C^{*}$-algebras of endomorphisms of groups with finite cokernel and partial actions, arXiv:1707.03056, (2017).

282. L. Zhang, R. Niu, Maschke-type theorem for partial smash products, Int. Electron. J. Algebra, 19, (2016), 49-57.

283. Y. Zhu, Some fundamental properties of tiling semigroups, J. Algebra, 252, (2002), 195-204.

Instituto de Matemática e Estatística, Universidade de SÃo Paulo, 05508090 SÃo PAUlo, SP, Brasil

E-mail address: dokucha@gmail.com 